\title{
ALMA-resolved salt emission traces the chemical footprint and inner wind morphology of VY Canis Majoris *
}

\author{
L. Decin ${ }^{1}$, A. M. S. Richards ${ }^{2}$, T. J. Millar ${ }^{3}$, A. Baudry ${ }^{4,5}$, E. De Beck ${ }^{6}$, W. Homan ${ }^{1}$, N. Smith ${ }^{7}$, \\ M. Van de Sande ${ }^{1}$, and C. Walsh ${ }^{8}$
}

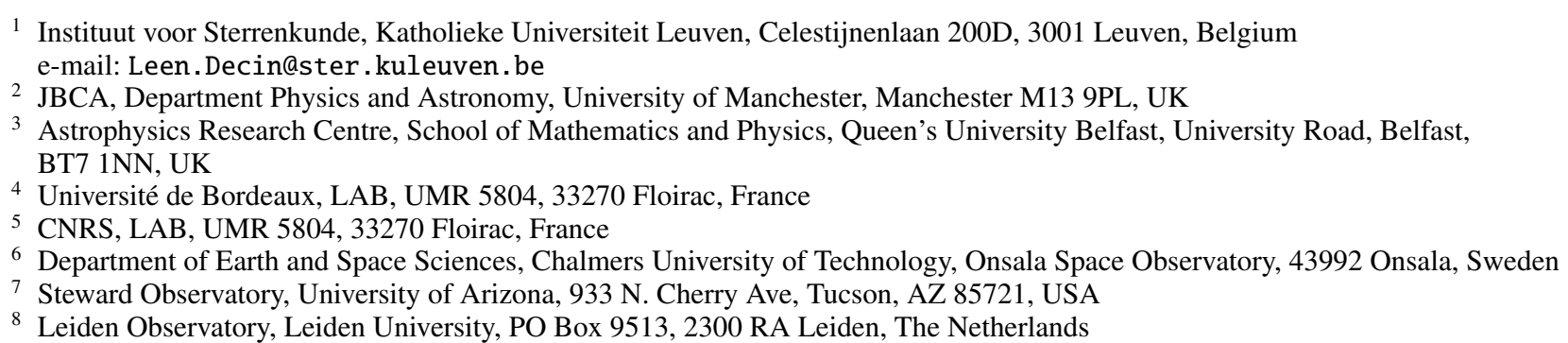

Received 9 December 2015 / Accepted 31 May 2016

\begin{abstract}
Context. At the end of their lives, most stars lose a significant amount of mass through a stellar wind. The specific physical and chemical circumstances that lead to the onset of the stellar wind for cool luminous stars are not yet understood. Complex geometrical morphologies in the circumstellar envelopes prove that various dynamical and chemical processes are interlocked and that their relative contributions are not easy to disentangle.

Aims. We aim to study the inner-wind structure $\left(R<250 R_{\star}\right)$ of the well-known red supergiant VY CMa, the archetype for the class of luminous red supergiant stars experiencing high mass loss. Specifically, the objective is to unravel the density structure in the inner envelope and to examine the chemical interaction between gas and dust species.

Methods. We analyse high spatial resolution $\left(\sim 0^{\prime \prime} 24 \times 0\right.$ '”13) ALMA science verification (SV) data in band 7 , in which four thermal emission lines of gaseous sodium chloride $(\mathrm{NaCl})$ are present at high signal-to-noise ratio.

Results. For the first time, the $\mathrm{NaCl}$ emission in the inner wind region of VY CMa is spatially resolved. The ALMA observations reveal the contribution of up to four different spatial regions. The $\mathrm{NaCl}$ emission pattern is different compared to the dust continuum and $\mathrm{TiO}_{2}$ emission already analysed from the ALMA SV data. The emission can be reconciled with an axisymmetric geometry, where the lower density polar/rotation axis has a position angle of $\sim 50^{\circ}$ measured from north to east. However, this picture cannot capture the full morphological diversity, and discrete mass ejection events need to be invoked to explain localized higher-density regions. The velocity traced by the gaseous $\mathrm{NaCl}$ line profiles is significantly lower than the average wind terminal velocity, and much slower than some of the fastest mass ejections, signalling a wide range of characteristic speeds for the mass loss. Gaseous $\mathrm{NaCl}$ is detected far beyond the main dust condensation region. Realising the refractory nature of this metal halide, this hints at a chemical process that prevents all $\mathrm{NaCl}$ from condensing onto dust grains. We show that in the case of the ratio of the surface binding temperature to the grain temperature being $\sim 50$, only some $10 \%$ of $\mathrm{NaCl}$ remains in gaseous form while, for lower values of this ratio, thermal desorption efficiently evaporates $\mathrm{NaCl}$. Photodesorption by stellar photons does not seem to be a viable explanation for the detection of gaseous $\mathrm{NaCl}$ at $220 R_{\star}$ from the central star, so instead, we propose shock-induced sputtering driven by localized mass ejection events as an alternative.

Conclusions. The analysis of the $\mathrm{NaCl}$ lines demonstrates the capabilities of ALMA to decode the geometric morphologies and chemical pathways prevailing in the winds of evolved stars. These early ALMA results prove that the envelopes surrounding evolved stars are far from homogeneous, and that a variety of dynamical and chemical processes dictate the wind structure.
\end{abstract}

Key words. supergiants - stars: mass-loss - circumstellar matter - stars: individual: VY Canis Majoris - astrochemistry instrumentation: interferometers

\section{Introduction}

The post-main-sequence evolution of stars with an initial mass in the range of $20-40 M_{\odot}$ may lead through a cool red supergiant (RSG) phase with strong mass loss. These stars can potentially evolve through a Wolf-Rayet phase as well if mass loss is sufficient to remove the hydrogen envelope and, if not, they may

\footnotetext{
* The datacubes (FITS files) are only available at the CDS via anonymous ftp to cdsarc.u-strasbg. fr (130.79.128.5) or via http://cdsarc.u-strasbg.fr/viz-bin/qcat?]/A+A/592/A76
}

explode as a supernova (Smith et al. 2009). The amount and nature of the mass loss dictates the future evolution of the star and determines their supernova ( $\mathrm{SN}$ ) appearance, be it as an $\mathrm{SN}$ of type II-P, II-L, IIb, IIn, or Ib/c. If a dense circumstellar envelope is still present at the moment of explosion, it will provide an obstacle for the SN blast wave to overtake (Pun et al. 2002).

Currently the driving mechanism of the mass loss of RSGs is not yet understood. Mechanisms based on radiation pressure on freshly synthesized dust grains, magneto-acoustic waves, and turbulent pressure owing to convection in combination 
with radiative pressure on molecular lines (Josselin \& Plez 2007) are invoked, or a combination of the above in a socalled hybrid model featuring magneto-rotational effects with a dust driven-wind scenario (Thirumalai \& Heyl 2012). Not being able to constrain the driving mechanism(s) represents a serious limitation for current stellar evolution models, which therefore adopt a simplified parametrized, time-averaged, massloss rate prescription (e.g. Reimers 1975; de Jager et al. 1988; Nieuwenhuijzen \& de Jager 1990). Empirical studies, however, show that the mass loss rate, $\dot{M}(t)$, may vary significantly during the RSG evolution (e.g. Decin et al. 2006) and that these stars can display sudden episodic bursts of mass loss that is potentially linked to the magnetic and/or convective nature of the star (Smith et al. 2001; Adande et al. 2013).

The circumstellar envelopes (CSEs) created by the intense mass loss are rich chemical laboratories in the Universe. Because of the low temperatures in the envelopes and the long duration of the mass loss, molecules and solid-state species are formed in these CSEs. Through stellar winds, these elements are injected into the interstellar medium (ISM), thereby gradually enriching their surroundings.

Efforts to deepen our understanding of the origin of the massloss and of the thermodynamical and chemical behaviour of the CSE proceed on two separate tracks: more detailed studies of specific RSGs which are often selected by virtue of their proximity, and global studies of a larger sample of RSGs to derive general characteristics. The first of these approaches is pursued in this paper, in which we present ALMA high spatial resolution $\left(\sim 0 \prime\right.$ !24 $\times 00^{\prime \prime}$ 13) observations of the close-by RSG VY CMa in thermal sodium chloride $(\mathrm{NaCl})$ emission lines, tracing the inner wind region within $\sim 1.5^{\prime \prime}$ ( or $\sim 280 R_{\star}$ ) from the central star.

\subsection{The red supergiant $V Y C M a$}

VY CMa is one of the most intrinsically luminous red stars in the galaxy $\left(L \sim 3 \times 10^{5} L_{\odot}\right.$; Smith et al. 2001; Choi et al. 2008). Its current mass is estimated around $17 M_{\odot}$, but initially it was some $25 M_{\odot}$ (Wittkowski et al. 2012). VY CMa is also known to be one of the largest RSGs with a stellar radius, $R_{\star}$, of $1420 \pm 120 R_{\odot}$ (Wittkowski et al. 2012) or 5.53 mas at a distance of $1.2 \pm 0.1 \mathrm{kpc}$ (Choi et al. 2008; Zhang et al. 2012). The average recent mass-loss rate is high, around $1-4 \times 10^{-4} M_{\odot} \mathrm{yr}^{-1}$ (Danchi et al. 1994; Monnier et al. 1999; Decin et al. 2006), producing a large circumstellar nebula some $10^{\prime \prime}$ (or $0.06 \mathrm{pc}$ ) across (Humphreys et al. 2007; Muller et al. 2007). The stellar velocity estimated from $\mathrm{OH} 1612 \mathrm{MHz}$ and $\mathrm{SiO}$ maser observations is $22 \pm 1 \mathrm{~km} \mathrm{~s}^{-1}$ (Richards et al. 1998) (all velocities are with respect to the local standard of rest, LSR).

Optical and near-infrared high-resolution imaging and interferometry have revealed a complex distribution of knots and filamentary arcs in the asymmetric reflection nebula around the obscured central star (Kastner \& Weintraub 1998; Monnier et al. 1999; Smith et al. 2001). Images, polarimetric data and radial velocity measurements (Smith 2004; Humphreys et al. 2007; Jones et al. 2007) show evidence that the arcs and knots in the ejecta are not only spatially distinct features, but are also kinematically separate from the surrounding diffuse material representing the steady wind (Humphreys et al. 2007; Smith et al. 2009). The current understanding is that VY CMa went through a series of episodic mass ejections with dense cloudlets moving at $\sim 35 \mathrm{~km} \mathrm{~s}^{-1}$ that produced a very high mass-loss rate of $\sim 2 \times 10^{-3} M_{\odot} \mathrm{yr}^{-1}$ between a few hundred and $1000 \mathrm{yr}$ ago. The diffuse halo might expand at a slightly lower speed $\left(\sim 25 \mathrm{~km} \mathrm{~s}^{-1}\right)$ and corresponds to the current average mass-loss rate of $\sim 3 \times 10^{-4} M_{\odot} \mathrm{yr}^{-1}$ (Smith et al. 2009). Optical and infrared data also provide some evidence for a disk-like geometry near the central object (Herbig 1970, 1972; McCarthy 1979; Efstathiou \& Rowan-Robinson 1990). The derived geometry is not always consistent between these studies. $K$-band interferometric measurements indicate a dusty disk oriented east-west (Monnier et al. 2004), but infrared images have been interpreted as evidence for a northeast-southwest axis of symmetry that represents the polar/rotation axis of the star and disk, which is tilted by $15^{\circ}$ to $30^{\circ}$ to our line of sight (for an overview, see Smith et al. 2001; Humphreys et al. 2007). Maser data have been interpreted as an expanding outflow, which at sub-arcsecond scale is more extended in the east-northeast and west-southwest direction (Morris \& Bowers 1980; Bowers et al. 1983; Richards et al. 1998). The idea of a preferred northeast-soutwest axis is roughly supported by the Hubble Space Telescope (HST) data of Smith et al. (2001) of which the asymmetric emission in the South-West could be interpreted as being reflection along the lower density bipolar axis oriented northeast-southwest with the south-western lobe closer to us (see also the cartoon of the proposed geometry in Fig. 13 in Smith et al. 2009). The apparent random orientation of the arcs, filaments and knots in the wind of VY CMa suggest that they were produced by localized mass ejections, not necessarily aligned with either the star's presumed northeast-southwest axis or its equator, and hence independent of any proposed bipolar geometry.

While data taken at optical and infrared continuum wavelength bands are prone to high extinction effects complicating an unambiguous interpretation of the inner wind structure of VY CMa, the scattering and extinction in the far-infrared and submillimeter domain are almost negligible. The retrieval of the morphological, kinematical and chemical structure of the inner wind region from prior observations was often hampered by a lack of spatial resolution to resolve the different kinematical components. Another difficulty was the association of molecular line emission to specific morphological features which was only based on a tentative interpretation of spatially unresolved velocity features in the broad asymmetric line profiles (Ziurys et al. 2007; Milam et al. 2007; Adande et al. 2013). Modern interferometers offer an angular resolution comparable to the angular scale of some arcs and knots in the CSE of VY CMa and interferometric far-infrared and submillimeter observations of molecular lines hence provide us with crucial diagnostics to unravel the physical and chemical processes shaping the inner envelope of VY CMa. With the aim to investigate the spatio-kinematical structure of the nebula around VY CMa, Kamiński et al. (2013b) have used the Submillimeter Array (SMA) to undertake an interferometric spectral imaging survey between 279 and $355 \mathrm{GHz}$ at a spatial resolution of $0.9^{\prime \prime}$. Twenty-one molecules were detected, of which 11 show a "point-like" morphology, 4 were indicated as "double" with a second source located to the southwest of the star (at 0 "'8 to 1 !' 2 ), one molecule $(\mathrm{CN})$ has a "ring-like" distribution, and 5 showed an "extended" distribution with multiple components. One of the detected molecules is $\mathrm{NaCl}$, showing a "point-like" angular distribution in the SMA data with the exception of the most intense $\mathrm{NaCl}$ line which shows weak emission in the southwest clump.

VY CMa was observed with ALMA during science verification in band 7 and band 9 (Richards et al. 2014). Four $\mathrm{NaCl}$ thermal emission lines are detected in the band 7 data, and are spatially resolved at an angular resolution of $\sim 0^{\prime \prime} .24 \times 0$ 0'.13 (see Sect. 2). The benefits of studying the spatio-kinematical and chemical structure of the nebula around VY CMa via 
Table 1. $\mathrm{NaCl}$ lines in the spectral windows covered by the ALMA observations.

\begin{tabular}{|c|c|c|c|c|c|c|}
\hline Transition & $\begin{array}{c}v_{\mathrm{lab}} \\
{[\mathrm{GHz}]}\end{array}$ & $\begin{array}{c}E_{\text {low }} \\
{\left[\mathrm{cm}^{-1}\right]}\end{array}$ & $\begin{array}{c}A \\
\mathrm{~s}^{-1}\end{array}$ & $\begin{array}{c}\text { Rms noise } \\
{\left[\mathrm{mJy} \mathrm{beam}^{-1} \mathrm{~km} \mathrm{~s}^{-1}\right]}\end{array}$ & $\begin{array}{c}\text { Beam } \\
{[“ \times ”, P A]}\end{array}$ & Comment \\
\hline$v=1 J=24-23$ & 309.787 & 480.09 & 0.017 & 19 & $0.232 \times 0.131,28.27^{\circ}$ & unblended \\
\hline$v=0 J=24-23$ & 312.110 & 119.83 & 0.013 & 30 & $0.239 \times 0.134,29.48^{\circ}$ & unblended \\
\hline$v=1 J=25-24$ & 322.650 & 490.42 & 0.019 & 27 & $0.222 \times 0.126,27.55^{\circ}$ & red wing slightly blended by $\mathrm{TiO}_{2}$ line \\
\hline$v=0 J=25-24$ & 325.069 & 130.24 & 0.015 & 195 & $0.217 \times 0.119,29.27^{\circ}$ & $\begin{array}{l}\text { blue wing blended by } \mathrm{H}_{2} \mathrm{O} \text { maser } \\
+ \text { blend with } \mathrm{SiS} v=1 J=18-17\end{array}$ \\
\hline
\end{tabular}

Notes. Listed are the transition, rest frequency, lower state energy, Einstein $A$-values from CDMS, the rms noise, the synthesized beam, and comments concerning line blending.

$\mathrm{NaCl}$ emission are manifold: (1) $\mathrm{NaCl}$ is a diatomic molecule with a simple Grotrian-diagram reducing the complexity of radiative transfer analysis and thus helping to retrieve the morphology of the nebula; (2) $\mathrm{NaCl}$ has a high dipole moment $(\mu=9 \mathrm{D}$, Müller et al. 2005) enabling efficient radiative excitation and hence providing a link between the line emission and the radiation field throughout the nebula; (3) while the abundance of $\mathrm{NaCl}$ in the wind of VY CMa is estimated to be some 5 orders of magnitude lower than that of CO (Milam et al. 2007), its high dipole moment facilitates its detection; and (4) as most metalbearing molecules $\mathrm{NaCl}$ is very refractory and is expected to quite easily form solid-state condensates so that one can investigate the gas-grain chemistry in the envelope. Understanding the gas-grain chemistry in this kind of oxygen-rich winds has a very broad application, since the abundances of the most chemically active species are quite close to the Galactic cosmic abundances and hence provides insight in the chemical processes at work on broad galactic scales.

In Sect. 2 we describe the ALMA observations and some details of the data reduction. Section 3 provides a detailed description of the spectral line profiles, the spatial distribution of the $\mathrm{NaCl}$ emission seen in the channel maps and a non-exhaustive comparison to other tracers of the morphological structure not yet mentioned in the introduction. In Sect. 4 we discuss the $\mathrm{NaCl}$ excitation conditions, construct a simplified chemical network aiming at unravelling the cycling between gaseous and solid $\mathrm{NaCl}$ (Sect. 4.1), and finally embark on an in-depth discussion on the morphology of the inner envelope based on the ALMA NaCl channel maps (Sect. 4.2). We summarize our conclusions in Sect. 5.

\section{Observations}

VY CMa was observed for ALMA science verification on 2013 16-19 August using 16-20 12-m antennas on baselines from $0.014-2.7 \mathrm{~km}$. The main objective was to map the $\mathrm{H}_{2} \mathrm{O}$ maser lines at 321 and $325 \mathrm{GHz}$ (Band 7) and $658 \mathrm{GHz}$ (Band 9), but several thermal lines identified with various rotational transitions of $\mathrm{NaCl}, \mathrm{TiO}_{2}, \mathrm{SO}_{2}$, and $\mathrm{SiO}$ were also present in the spectral setting in addition to the continuum data. Richards et al. (2014) summarises the observations, data reduction and $\mathrm{H}_{2} \mathrm{O}$ maser results, while O'Gorman et al. (2015) describes the dust-dominated continuum in more detail. The $\mathrm{TiO}_{2}$ thermal emission lines are presented by De Beck et al. (2015). Two prominent components are visible in the continuum dust emission around $321 \mathrm{GHz}$. The brightest dust component "C" has a peak position at $\alpha=07 \mathrm{~h} 22 \mathrm{~m} 58.34293 \mathrm{~s}$ and $\delta=-25^{\circ} 46^{\prime} 03^{\prime \prime}$.2227, while the position of the peak of the second continuum component has been shown to coincide with the location of the stellar position "VY" at $\alpha=07 \mathrm{~h} 22 \mathrm{~m} 58.326 \mathrm{~s}$ and $\delta=-25^{\circ} 46^{\prime} 03^{\prime \prime}$.042 (Richards et al. 2014; O'Gorman et al. 2015) (see Fig. 2, Fig. 7).

Four $\mathrm{NaCl}$ lines in the ground or first vibrational state are covered in the ALMA band 7 data $^{1}$. All of them are detected, albeit only two are unblended (see Table 1). The $\mathrm{NaCl} v=1$ $J=25-24$ is slightly blended with the $\mathrm{TiO}_{2}\left(33_{8,26}-33_{7,27}\right)$ line at $322.613 \mathrm{GHz}$ (De Beck et al. 2015), while the $\mathrm{NaCl} v=0$

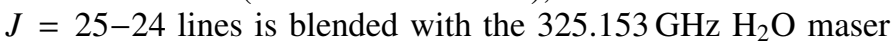
line (Richards et al. 2014) and the $\operatorname{SiS} v=1 J=18-17$ line at $325.059 \mathrm{GHz}$ (see Sect. 3.1). No rotational lines in the ground or first vibrational state of the minor isotopologue $\mathrm{Na}^{37} \mathrm{Cl}$ were covered in the current ALMA spectral setup in band 7. The $\mathrm{NaCl}$ $v=3 J=51-50$ line at $657.134 \mathrm{GHz}$ was also detected, but is severely blended with the much stronger $\mathrm{H}_{2} \mathrm{O}$ maser line at $658 \mathrm{GHz}$, making a quantitative analysis impossible and hence it is not further discussed.

The synthesized beamsize for the band 7 data is $\sim 0$ '. $24 \times$ $0 \prime \prime 13$ (see Table 1). The stellar continuum was detectable in the individual channels at all frequencies, so that the relative alignment of all lines is good to $\sim 2$ mas (Richards et al. 2014). The continuum was subtracted before making the final line cubes. All data were adjusted to constant velocity with respect to the Local Standard of Rest.

Image cubes were made at $1 \mathrm{~km} \mathrm{~s}^{-1}$ spectral resolution around the $\mathrm{NaCl}$ frequencies listed in Table 1 . The higher $\sigma_{\text {rms }}$ noise around $325 \mathrm{GHz}$ is due to the poor atmospheric transmission in this region. The noise in the brightest channels is slightly higher in all cases due to dynamic range limitations. The shortest baseline was $14 \mathrm{~m}$ and inspection of the continuum visibility amplitudes against baseline length shows that the flux density remains quite steady out to $170 \mathrm{~m}$ at $321-325 \mathrm{GHz}$, suggesting that we recover all the flux on scales $<13^{\prime \prime}$, with reliable imaging on scales up to at least $8^{\prime \prime}$. The absolute astrometric accuracy of the lines described here is 35 mas and the flux density scale is accurate to at least $10 \%$.

\section{Results}

\subsection{Spectral line profiles}

Figure 1 shows the flux densities extracted for a $1^{\prime \prime}$ diameter region around the stellar position "VY" for the four $\mathrm{NaCl}$ lines; no $\mathrm{NaCl} v=1$ emission is detected beyond this aperture. The four $\mathrm{NaCl}$ lines are centred around the $v_{\mathrm{LSR}}$ of $\sim 22 \mathrm{~km} \mathrm{~s}^{-1}$ (Richards et al. 1998, 2014; Tenenbaum et al. 2010; Kamiński et al. 2013b) and show similar line shapes ranging from $\sim 6 \mathrm{~km} \mathrm{~s}^{-1}$ to $\sim 40 \mathrm{~km} \mathrm{~s}^{-1}$. Given the similar energy levels,

\footnotetext{
1 The identification of the $\mathrm{NaCl}$ lines is based on the Cologne Database for Molecular Spectroscopy (CDMS; Müller et al. 2001, 2005).
} 


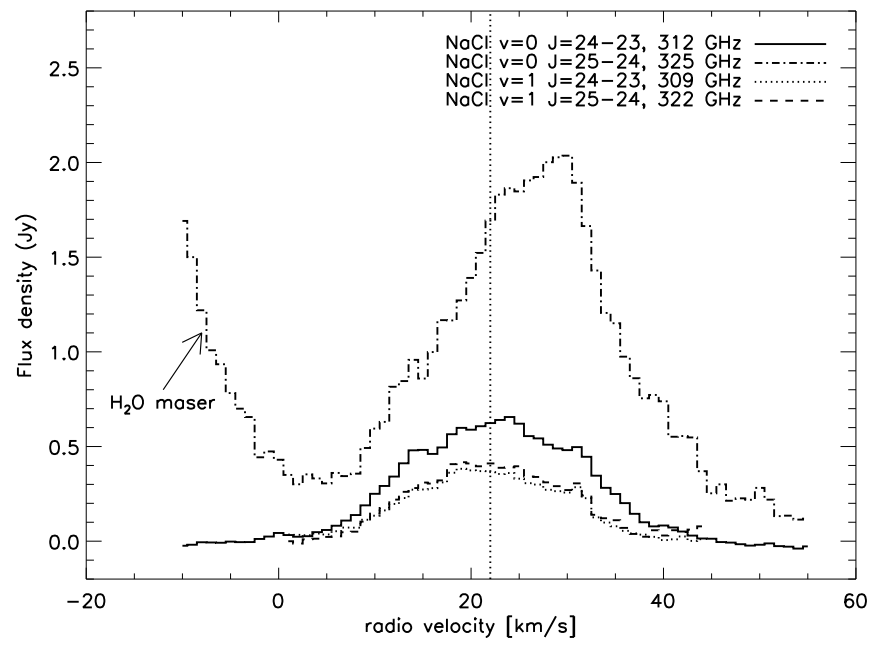

Fig. 1. Flux densities for the four $\mathrm{NaCl}$ lines detected in the ALMA band 7 data. Flux densities are for an aperture with circular diameter of 1 " centred at "VY". The LSR velocity of $22 \mathrm{~km} \mathrm{~s}^{-1}$ is indicated by the vertical dotted line. Line blending causes the flux density for the $325 \mathrm{GHz} \mathrm{NaCl} v=0 \mathrm{~J}=25-24$ line to be skewed and to be too high with an expected contribution of the $\operatorname{SiS} v=1 J=18-17$ line of $\sim 1.1 \mathrm{Jy}$ (see text for more details).

quantum numbers, and Einstein-A coefficients, one would expect similar line intensities and spatial distributions for both lines in the same vibrational level. While this holds for the $\mathrm{NaCl}$ lines in the first vibrational level, the $325 \mathrm{GHz} \mathrm{NaCl} J=25-24$ line in the vibrational ground state is $\sim 1.4 \mathrm{Jy}$ higher than the $312 \mathrm{GHz}$ $\mathrm{NaCl} v=0 J=24-23$ peak. This appears to be due to blending. The $325.153 \mathrm{GHz}$ water maser (with a $\sim 500 \mathrm{Jy}$ peak) has a very broad profile, with a red-shifted wing $>70 \mathrm{~km} \mathrm{~s}^{-1}$ and contributes roughly $0.2 \mathrm{Jy}$ to the low-velocity wing of the $325.069 \mathrm{GHz}$ $\mathrm{NaCl}$ line. The $325.059 \mathrm{GHz} \mathrm{SiS} v=1 J=18-17$ line is even closer to the $\mathrm{NaCl}$ line (peaks separated by $9.2 \mathrm{~km} \mathrm{~s}^{-1}$ ). To estimate the line strength of the SiS $v=1 J=18-17$, we use the results of Kamiński et al. (2013b) who made a spectral line survey of VY CMa between 279 and $355 \mathrm{GHz}$ using the SMA. They were unable to detect the $325.059 \mathrm{GHz}$ SiS line due to the poor atmospheric transmission but, using a $1^{\prime \prime}$ radius aperture, they measured maximum flux densities of $0.96 \mathrm{Jy}$ for the $\mathrm{SiS}$ $v=1 J=16-15$ line and $0.97 \mathrm{Jy}$ for the $J=17-16$ line. The estimated maximum flux for the $\operatorname{SiS} v=1 J=19-18$ is indicated to be uncertain, with a value around $1.38 \mathrm{Jy}$. From these results, we estimate that the $\operatorname{SiS} v=1 J=18-17$ line can contribute $\sim 1.1$ Jy to the flux density profile of the $325 \mathrm{GHz}$ $\mathrm{NaCl}$ line, mostly on the higher-velocity side. This explains why this line is skewed to the red-shifted side of the stellar velocity. If $1.1-1.3 \mathrm{Jy}$ is subtracted from the apparent $325 \mathrm{GHz} \mathrm{NaCl}$ line profile, the remaining peak would be close to the $0.6 \mathrm{Jy}$ peak of the $312 \mathrm{GHz} \mathrm{NaCl}$ line. After correction for line blending, both $v=1$ lines have flux densities within a factor of $\sim 2$ of the $v=0$ lines despite an energy separation of $\sim 360 \mathrm{~cm}^{-1}$, which is caused by the dominantly radiative excitation mechanism.

\subsection{Spatial distribution}

We created moment-zero maps (total intensity) for each of the $\mathrm{NaCl}$ lines. The $\mathrm{NaCl}$ lines in a given vibrational level show similar spatial distributions. Since the $\mathrm{NaCl} J=25-24$ lines are blended in both vibrational levels, we discuss the spatial distribution of $\mathrm{NaCl}$ on the basis of the $J=24-23$ rotational lines; the channel maps for the $\mathrm{NaCl} J=25-24$ lines are shown in Appendix A. A zoom for the inner 0.8" around the stellar position "VY" is shown in Figs. 2 and 3. In this inner 0.8" region, the spatial distribution of both lines show a similar bimodal distribution with two clumps at small offsets from the stellar position. For most of the channel maps, the northern clump ("NB" from here onward) is brighter than the southern clump ("SB" from here onward); see also Fig. 5 for an indication of the nomenclature used in this paper. In addition, both rotational $\mathrm{NaCl}$ lines in the ground-vibrational state display a third emission component located at $\sim 1.2$ " to the South-West ("SW" clump); see Fig. 4.

For the $\mathrm{NaCl}$ lines in the first vibrational state, all the emission is concentrated in "NB" and "SB"; the other two lines in the ground vibrational state show two (one) "SW" component(s) at 312 (325) GHz. The two "SW" components for the $\mathrm{NaCl} v=0$ $J=24-23$ line at $312 \mathrm{GHz}$ are called "SW1" and "SW2" (see Fig. 5), with "SW1" being brighter than "SW2". We measured the sizes and positions of each spatial region by fitting two dimensional elliptical Gaussian components (see Table 2). The position uncertainties given are estimated conservatively from the noise as (mean beam size)/(signal to noise ratio) but do not include the 2 mas line-to-line alignment uncertainty (the 35 mas absolute astrometric uncertainty does not affect alignment of these $\mathrm{NaCl}$ lines). The component sizes are the FWHM (full width at half maximum) deconvolved from the beam where possible; only "NB" is consistently fully resolved and the errors given take into account that the sizes of the other components are in some cases upper limits.

The brightest "NB" component is aligned to within 8 mas in both coordinates at 309,312 and $322 \mathrm{GHz}$, which is within the 3 sigma position uncertainties. The fainter "SB" component is aligned to within 40 mas; although this could be significant it is more probably due to fitting uncertainties in the case of a nonGaussian flux distribution. The average separations of "NB" and "SB" from the stellar position, "VY", are 100 mas (or $\sim 18 R_{\star}$ ) and 190 mas (or $\sim 34 R_{\star}$ ), respectively. The separation between "NB" and "SB" is 265 mas $\left(\sim 48 R_{\star}\right)$. The relative uncertainty in each case is 10 mas $\left(\sim 2 R_{\star}\right)$. The brighter "SW" component, "SW1", seen at $312 \mathrm{GHz}$ is $1.22^{\prime \prime}\left(\sim 220 R_{\star}\right)$ from VY; the fainter "SW2" component is $0.3^{\prime \prime}$ further away. Formally, the angular separations are a lower limit to the physical separation, but since the $\mathrm{NaCl}$ clumps' velocities span the stellar velocity, these are probably close to the actual separations.

"NB" and "SB" and a single "SW" clump are seen in the $\mathrm{NaCl} v=0 J=25-24$ line at $325 \mathrm{GHz}$. The uncertainties listed in Table 2 are a few times greater, due both to the noise level which is 6-10 times higher and blending with the other lines, in particular with the water maser which is widely distributed around the "NB" and "SB" clumps and beyond but not towards the "SW" clump.

The northern clump is visible in the channel maps for all observed $\mathrm{NaCl}$ transitions with velocities between 6 and $37 \mathrm{~km} \mathrm{~s}^{-1}$. The flux density in "NB" shows two local maxima, one around $14 \mathrm{~km} \mathrm{~s}^{-1}$ ("NB blue") ) and the other around $24 \mathrm{~km} \mathrm{~s}^{-1}$ ("NB red"); see Fig. 6. Emission from "SB" arises in channel maps with velocities between 12 and $26 \mathrm{~km} \mathrm{~s}^{-1}$. The flux density of "SB" reaches a maximum around $19 \mathrm{~km} \mathrm{~s}^{-1}$, i.e. coinciding with a local minimum in the flux density of "NB". The 'SW' extension in the ground vibrational lines is visible for velocity channels between $12-33 \mathrm{~km} \mathrm{~s}^{-1}$ (see Fig. 4) with a maximum flux density around $25 \mathrm{~km} \mathrm{~s}^{-1}$ (see Fig. 6). 


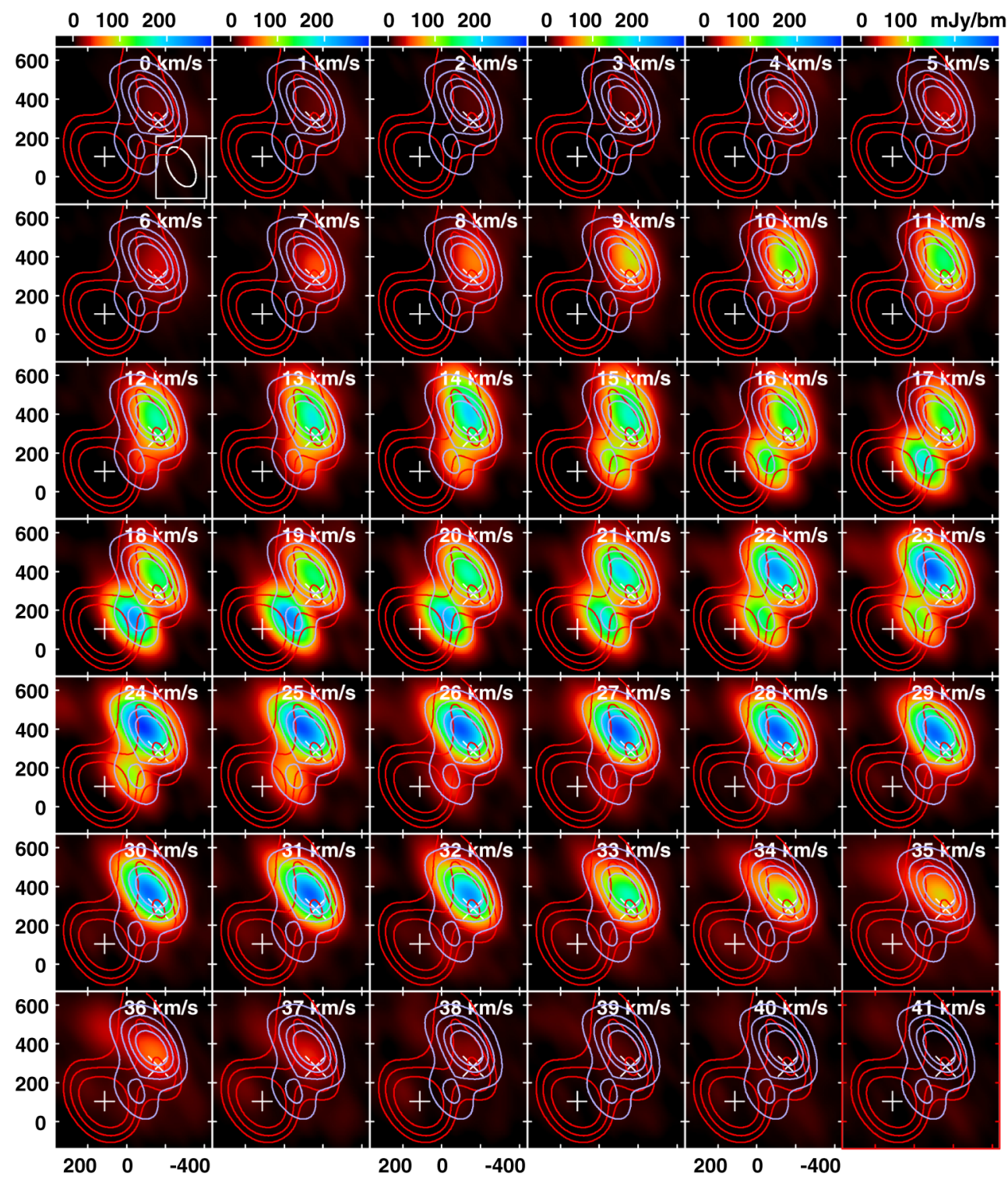

Fig. 2. Channel maps (colour scale) of the $\mathrm{NaCl} v=0 J=24-23$ emission at $312 \mathrm{GHz}$ at a $1 \mathrm{~km} \mathrm{~s}^{-1}$ velocity resolution for the central 0 "' $8 \times 0$ "'.8 field around "VY" and "C". Grey contours are the integrated $\mathrm{NaCl}$ line strength at $(1,2,3,4) \times 1 \mathrm{Jy} / \mathrm{beam} \mathrm{km} \mathrm{s}^{-1}$, red contours are the dust continuum measured with ALMA at $321 \mathrm{GHz}$ at $(1,2,3,4) \times 18 \mathrm{mJy} /$ beam (Richards et al. 2014; O'Gorman et al. 2015). The stellar position "VY" is indicated with a white cross, and the peak of the continuum emission "C" is indicated with a white plus-sign. The ordinate and co-ordinate axis give the offset of the right ascension and declination, respectively, in units of milli-arcseconds.

\subsection{Morphological comparison to other tracers of the envelope}

In this section we compare the $\mathrm{NaCl}$ emission seen in the ALMA channel maps to some other results presented in the literature. We only focus on observational results already retrieved from ALMA data and on studies which discuss the $\mathrm{NaCl}$ emission and/or the extension seen at $1.2^{\prime \prime}$ to the south-west.

The maximum known angular extent of the molecular envelope around VY CMa as traced by CO (Muller et al. 2007; Kamiński et al. 2013b) and $\mathrm{OH}$ maser emission (Richards 1997 ) is $\sim 10^{\prime \prime}$ and the typical velocity span is from -15 to $+60 \mathrm{~km} \mathrm{~s}^{-1}$, with several species observed closer to the central star showing a more extended red wing up to $+105 \mathrm{~km} \mathrm{~s}^{-1}$ (see, e.g. Kamiński et al. 2013b; De Beck et al. 2015). However, where imaging is available, these extreme velocities appear at only a few position angles, and most of the molecular shell appears to show radial acceleration out to a terminal velocity of $35-45 \mathrm{~km} \mathrm{~s}^{-1}$ (Decin et al. 2006; Muller et al. 2007; Ziurys et al. 2007) ${ }^{2}$. The $\mathrm{NaCl}$ emission detected by ALMA band 7 has hence a much more compact distribution and while its emission extends significantly beyond the estimated dust formation radius of 35-55 mas (Danchi et al. 1994; Monnier et al. 1999; Smith et al. 2001; Decin et al. 2006) it does not reach the terminal velocity.

Figures 2, 3, A.1, and A. 2 compare the $\mathrm{NaCl}$ emission to the dust emission detected in the ALMA band 7 band. The emission of the brightest $\mathrm{NaCl}$ components ("NB" and "SB") and the dust shows a similar central "waist" along a NE-SW axis, albeit the peak position of the $\mathrm{NaCl}$ clumps is to the north of "VY" and NW of " $\mathrm{C}$ " and are clearly offset from the dust continuum peaks.

2 Note that Smith et al. (2009) mentioned a terminal velocity for the diffuse cloud representing the steady wind of $25 \mathrm{~km} \mathrm{~s}^{-1}$; see Sect. 1 . 
$\begin{array}{lllllllllllllllllllllll}0 & 50 & 100 & 150 & 0 & 50 & 100150 & 0 & 50 & 100 & 150 & 0 & 50 & 100 & 150 & 0 & 50 & 100 & 150 & 0 & 50 & 100 \mathrm{mJy} / \mathrm{bm}\end{array}$

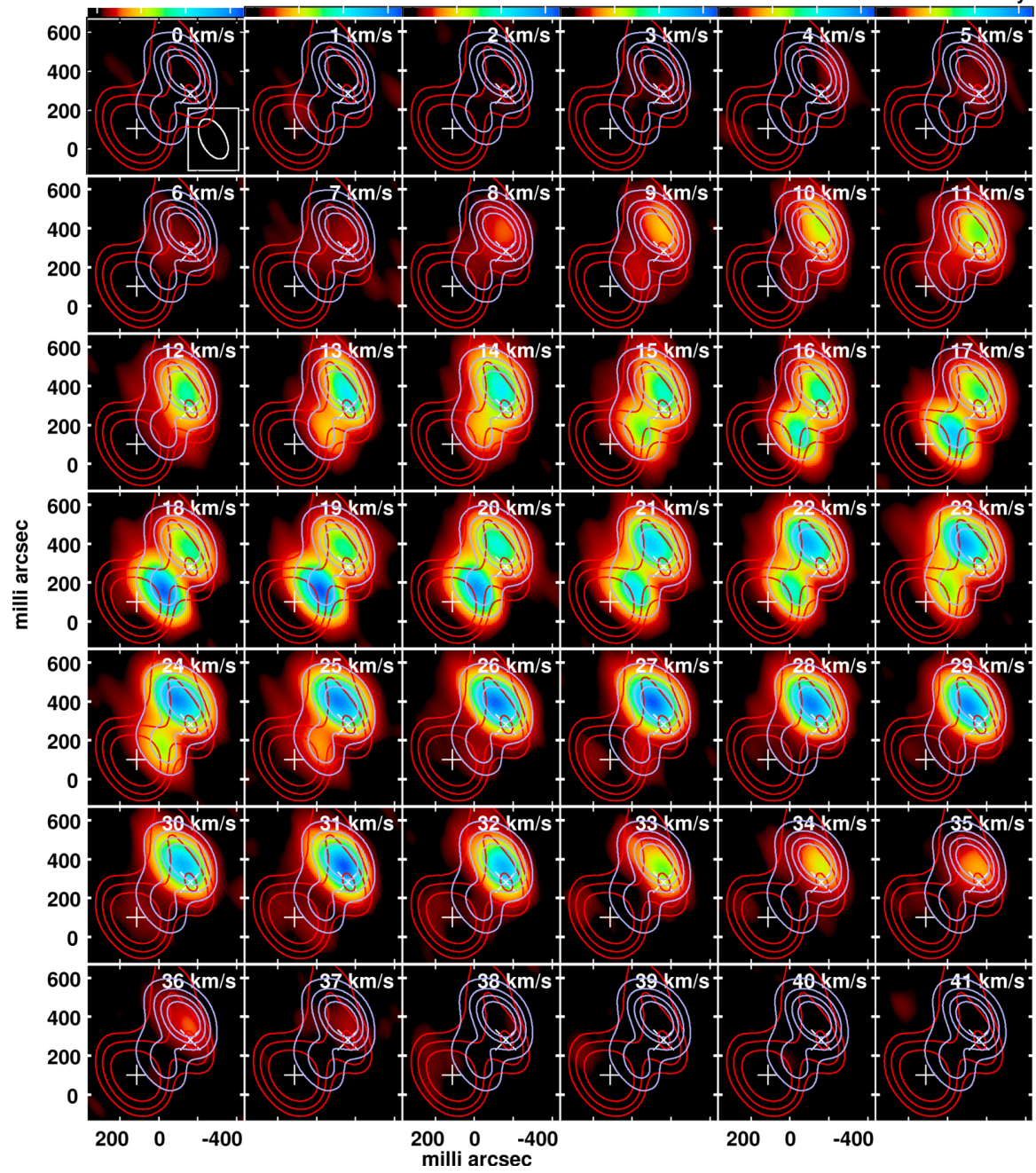

Fig. 3. Same as Fig. 2 but for the $\mathrm{NaCl} v=1$ $J=24-23$ line at $309 \mathrm{GHz}$.

Table 2. Information of the different spatial components seen in the ALMA $\mathrm{NaCl}$ channel maps.

\begin{tabular}{|c|c|c|c|c|c|c|c|c|c|c|}
\hline Region $^{a}$ & $\begin{array}{c}\text { Peak } \\
{\left[\text { Jy/beam km s }{ }^{-1}\right]}\end{array}$ & $\begin{array}{c}\text { Tot flux } \\
{\left[\mathrm{Jy} \mathrm{km} \mathrm{s}^{-1}\right]}\end{array}$ & $\begin{array}{c}\text { RA } \\
{[\mathrm{h}: \mathrm{m}: \mathrm{s}]}\end{array}$ & $\begin{array}{c}\text { Dec } \\
{\left[{ }^{\circ}::^{\prime \prime}\right]}\end{array}$ & $\begin{array}{c}\text { Pos err } \\
{[\mathrm{mas}]}\end{array}$ & $\begin{array}{l}\text { Maj ax } \\
{[\mathrm{mas}]}\end{array}$ & $\begin{array}{c}\operatorname{Min} a x \\
{[\mathrm{mas}]}\end{array}$ & $\begin{array}{l}\text { Size err } \\
{[\mathrm{mas}]}\end{array}$ & $\begin{array}{c}\text { PA } \\
\text { [degrees] }\end{array}$ & $\begin{array}{c}\text { PA err } \\
\text { [degrees] }\end{array}$ \\
\hline \multicolumn{11}{|c|}{$\mathrm{NaCl} v=1 J=24-23$ at $309 \mathrm{GHz}$} \\
\hline NB & 2.831 & 2 & 3 & - & 2 & 9 & 1 & 5 & 9 & \\
\hline SB & 1.248 & 2.144 & $07: 22: 5$ & -25 & 4 & 196 & & 10 & 18 & 10 \\
\hline \multicolumn{11}{|c|}{$\mathrm{NaCl} v=0 J=24-23$ at $312 \mathrm{GHz}$} \\
\hline NB & 5.327 & 10.539 & $07: 22: 5$ & $-25: 4$ & 2 & 213 & 141 & 35 & 50 & 45 \\
\hline & & & & & 4 & & & 7 & 63 & \\
\hline & & & $07: 22$ & $-25:$ & 7 & & & 16 & 9 & 160 \\
\hline SW2* & 0.856 & 1.860 & $07: 22: 58$ & $-25: 4$ & 9 & 276 & 131 & 200 & 42 & 18 \\
\hline \multicolumn{11}{|c|}{$\mathrm{NaCl} v=1 J=25-24$ at $322 \mathrm{GHz}$} \\
\hline NB & 2.883 & 6.295 & 0 & -2 & 3 & 181 & 166 & 7 & 85 & 20 \\
\hline SB $^{*}$ & 1.377 & 1.855 & $07: 22: 58$ & $-25: 4$ & 5 & 167 & 12 & 13 & 57 & 30 \\
\hline \multicolumn{11}{|c|}{$\mathrm{NaCl} v=0 J=25-24$ at $325 \mathrm{GHz}^{b}$} \\
\hline & 13.924 & 55.2 & & & 3 & 328 & & 2 & 156 & 12 \\
\hline & & & 0 & $-25: 4$ & 26 & 2 & & 26 & 61 & 18 \\
\hline SW & 0.588 & 1.843 & $07: 22: 58.2712$ & $-25: 46: 04.271$ & 72 & 292 & 150 & 200 & 144 & 180 \\
\hline
\end{tabular}

Notes. The first column indicates which spatial component was fitted with a 2-dimensional (2D) Gaussian function, the second column gives the peak of the fitted function, the third column the total flux, the fourth, fifth, and sixth column the right ascension, declination, and positional uncertainty of the center of the elliptical Gaussian fit, the seventh, eight and ninth column the major and minor axis of the deconvolved 2D Gaussian fit with the uncertainty, and the last two columns list the position angle and its uncertainty of the major axis. ${ }^{(a)}$ Indication of the different spatial regions (see Fig. 4). $\mathrm{A}^{(*)}$ denotes that the region is only partly resolved; i.e. the deconvolved size is an upper limit. ${ }^{(b)}$ Strongly blended with $\mathrm{H}_{2} \mathrm{O}$ and SiS line. 


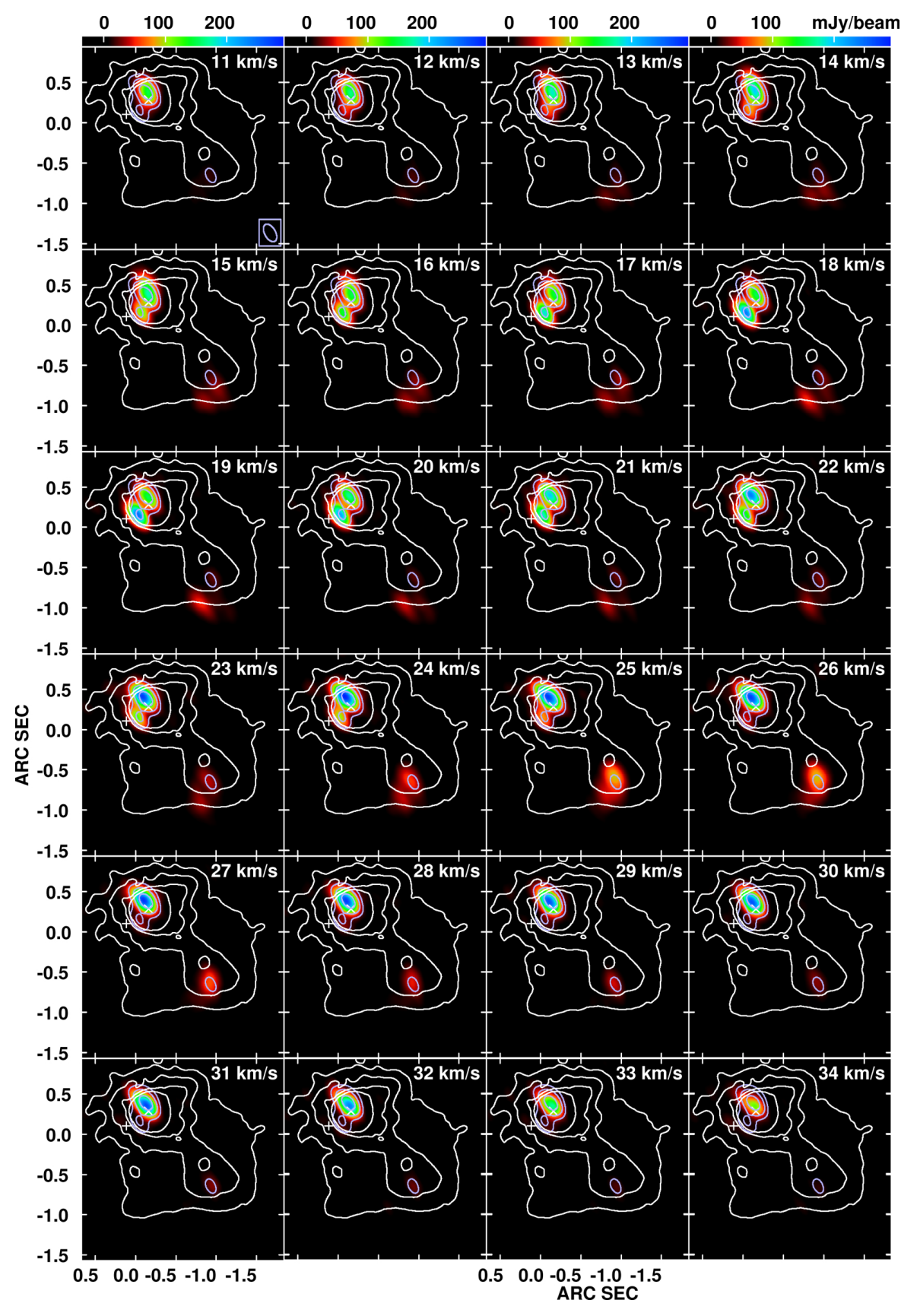

Fig. 4. Same as Fig. 2, but showing the central $2^{\prime \prime} .5 \times 2$ '.5 region. The white contours show the HST/WFPC2 data in the $F 1042 M$ filter around $1.018 \mu \mathrm{m}$ at $(1,2,4,8,16) \times 110$ counts (Smith et al. 2001).
As shown by Richards et al. (2014) the $\mathrm{H}_{2} \mathrm{O}$ 658, 321, and $325 \mathrm{GHz}$ maser emission cluster around the stellar position "VY", with another accumulation in the "valley" between "C" and "VY" (see Fig. 7). Richards et al. (1998) found the $22 \mathrm{GHz} \mathrm{H}_{2} \mathrm{O}$ masers to show a generally expanding envelope, with two "ears" outside the main $22 \mathrm{GHz}$ shell, which tentatively was interpreted as being suggestive for a biconical outflow. The south clump ("SB") of the $\mathrm{NaCl}$ emission lies in the "valley" between the two continuum peaks, to the south of some of the most intense water maser emission. The $22 \mathrm{GHz}$ and $321 \mathrm{GHz}$ $\mathrm{H}_{2} \mathrm{O}$ maser features at the greatest angular separations lie approximately east and west or to the southwest.

Milam et al. (2007) observed several rotational lines in the ground vibrational state of $\mathrm{NaCl}$ using single-dish telescopes with beam sizes between $29^{\prime \prime}-44^{\prime \prime}$. The $\mathrm{NaCl}$ emission was spatially unresolved and modelling the observed spectra resulted in an abundance relative to $\mathrm{H}_{2}$ of $5 \times 10^{-9}$ with a source size of $0.5^{\prime \prime}$. Kamiński et al. (2013b) observed several rotational lines of $\mathrm{NaCl}$ in vibrational states with $v \leq 3$ at an angular resolution of $\sim 0.9^{\prime \prime}$ using the SMA. The most intense lines were partially resolved, with the emission being slightly elongated with mean deconvolved FWHMs of $\left(0^{\prime \prime} .42 \pm 00^{\prime \prime} 05\right) \times$ $\left(0^{\prime \prime} 30 \pm 00^{\prime \prime} .04\right)$ and a position angle of $-33 \pm 2^{\circ}$. Weak emission in the SW clump at $\sim 1^{\prime \prime}$ is seen only in the most intense line of $\mathrm{NaCl}$ and could not be mapped with the SMA. Thanks to the high spatial resolution of ALMA, the $\mathrm{NaCl}$ emission is for the first time well resolved and a clear distinction can be made between emission seen in the bright northern clump, the slightly weaker southern clump, and the weak south-western extension (see Table 2 in Sect. 3.2), the latter only detected for the $\mathrm{NaCl}$ lines in the ground-vibrational state. Based on the $\mathrm{NaCl} v=0$ $J=24-23$ transition - the only unblended line for which the emission traces all the different spatial components - the northern clump has a deconvolved size of $\left(0^{\prime \prime} .213 \times 00^{\prime \prime} .141\right)$, the upper limit for the size of the southern clump is $\left(0^{\prime \prime} 138 \times 00^{\prime \prime} 134\right)$, while the two components in the south-western extension have sizes 


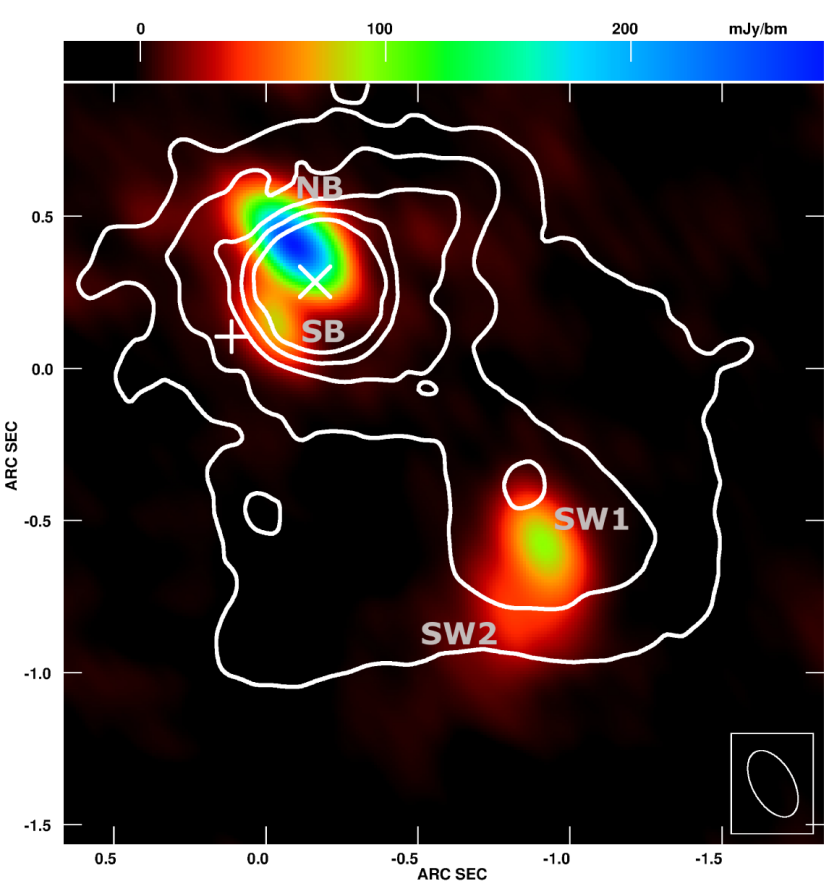

Fig. 5. ALMA $\mathrm{NaCl} 312 \mathrm{GHz}$ channel map at $25 \mathrm{~km} \mathrm{~s}^{-1}$ (colour scale) overlaid with the HST/WFPC2 F1042M data of Smith et al. (2001) (in contours at $(1,2,4,8,16) \times 110$ counts $)$. The cross sign indicates the position of the central star VY CMa, noted "VY" in the text, as derived from the ALMA $\mathrm{H}_{2} \mathrm{O}$ maser and continuum data (Richards et al. 2014; O'Gorman et al. 2015), the plus-sign indicates the maximum of the ALMA dust continuum data, noted as "C" in the text, (O'Gorman et al. 2015). The nomenclature of the different spatial regions refers to the northern clump 'NB', the southern clump 'SB', the brightest part of the south-western extension 'SW1', and the faintest part of the northwestern extension"SW2". The ordinate and co-ordinate axis give the offset of the right ascension and declination, respectively, in units of arcseconds.
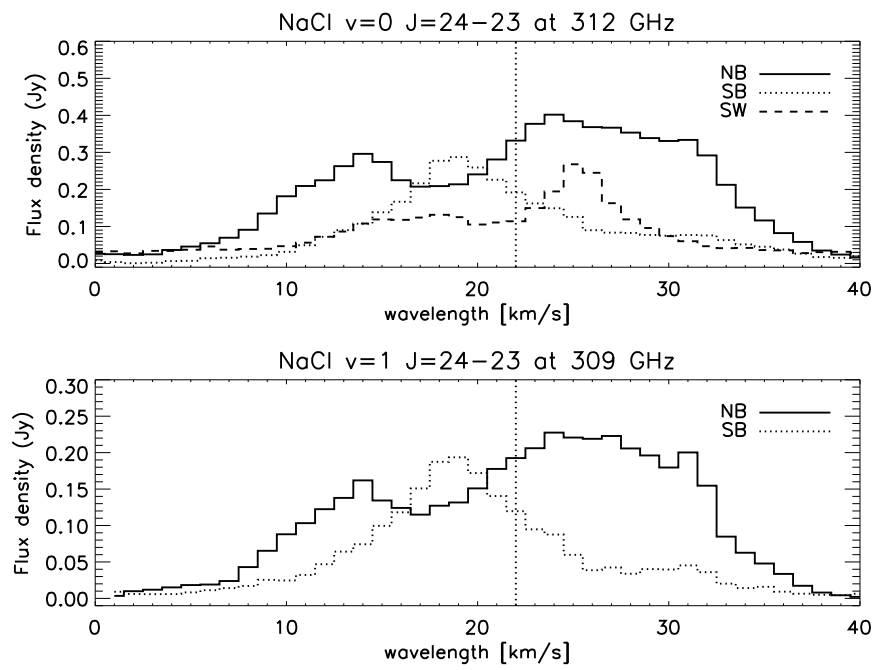

Fig. 6. Flux densities for the $\mathrm{NaCl} J=24-23$ lines in the groundvibrational state $v=0$ at $312 \mathrm{GHz}$ (upper panel) and in the first vibrational state $v=1$ at $309 \mathrm{GHz}$ (lower panel). Flux densities are extracted in the north clump (NB, full line) at $\left(07 \mathrm{~h} 22 \mathrm{~m} 58.326 \mathrm{~s},-25^{\circ} 46^{\prime} 02^{\prime \prime} .9\right)$ for an aperture of $\left(0^{\prime \prime} 394 \times 0^{\prime \prime} 373\right)$, in the south clump (SB, dotted line) at $\left(07 \mathrm{~h} 22 \mathrm{~m} 58.332 \mathrm{~s},-25^{\circ} 46^{\prime} 03^{\prime \prime} \cdot 118\right)$ for an aperture of $\left(0^{\prime \prime} 36 \times 0^{\prime \prime} \cdot 36\right)$, and in case of the $312 \mathrm{GHz}$ line in the south-west clump ( $\mathrm{SW}$, dashed line) at $\left(07 \mathrm{~h} 22 \mathrm{~m} 58.273 \mathrm{~s},-25^{\circ} 46^{\prime} 04^{\prime \prime} 028\right)$ for an aperture of $\left(0^{\prime \prime} .57 \times 0^{\prime \prime} \cdot 73\right)$. The LSR velocity of $22 \mathrm{~km} \mathrm{~s}^{-1}$ is indicated by the vertical dotted line.

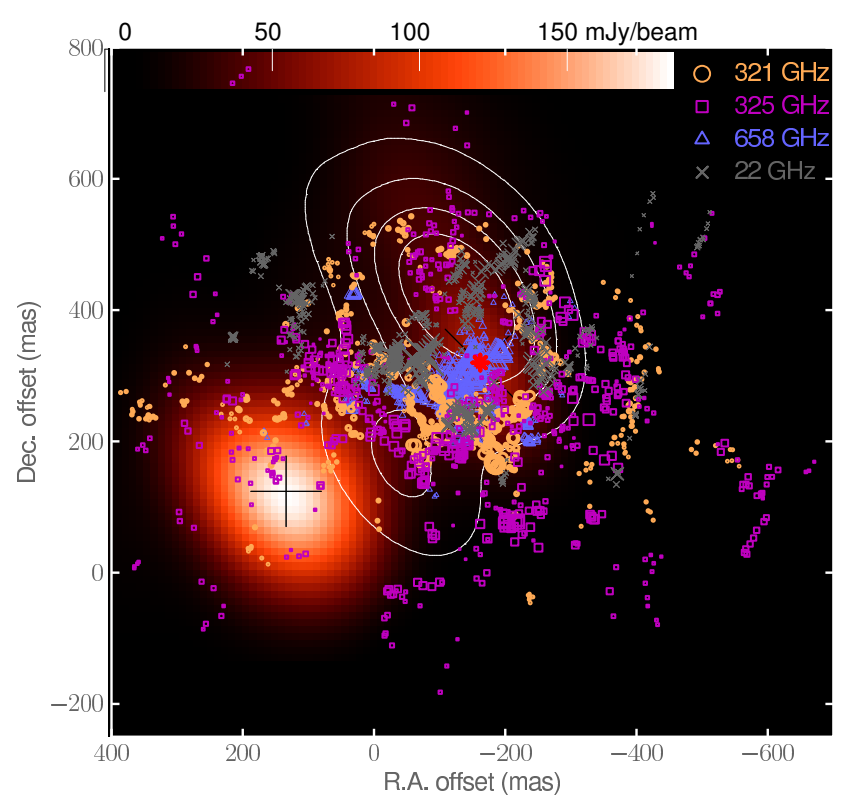

Fig. 7. The $\mathrm{H}_{2} \mathrm{O}$ maser emission (Richards et al. 1998, 2014) is shown on top of the $\mathrm{NaCl} 312 \mathrm{GHz}$ integrated line emission (gray contours, zeroth moment at $0.25,0.5,1,2,3,4 \mathrm{Jy} \mathrm{km} \mathrm{s}^{-1}$ ) and the ALMA dust continuum emission (colour scale; O'Gorman et al. 2015).

with upper limits being $\left(0^{\prime \prime} .281 \times 00^{\prime \prime} .174\right)$ and $\left(0^{\prime \prime} .276 \times 00^{\prime \prime} .131\right)$ (see Table 2).

A similar "SW" extension/clump at $\sim 1$ " for a position angle (PA) of $\sim 220^{\circ}$ w.r.t. "VY" is seen in some ALMA $\mathrm{TiO}_{2}$ channel maps (De Beck et al. 2015) and is also reported by Kamiński et al. (2013b) for some sulfur bearing species (CS, $\mathrm{NS}, \mathrm{SiS}$, and $\left.\mathrm{H}_{2} \mathrm{~S}\right)$ and the most intense $\mathrm{NaCl}(J=26-25)$ line. The line profile of the SMA $\mathrm{H}_{2} \mathrm{~S}$ and $\mathrm{CS}$ emission in the "SW" clump seen is rectangular with FWHM of $15 \mathrm{~km} \mathrm{~s}^{-1}$. The emission of this "SW" component very well matches the south-west extension seen in the Hubble Space Telescope (HST) data at wavelengths between $1-2.14 \mu \mathrm{m}$ that image the reflection nebula around VY CMa (see Fig. 5; Smith et al. 2001) and with other near-infrared and polarimetric data as for instance presented by Monnier et al. (1999) and Jones et al. (2007). The direction of this southwest extension/knot is roughly perpendicular to the possible density-enhanced equatorial plane as deduced from the one-sided reflection nebula seen in the visible and the maser elongation (see Sect. 1). More recently, Shenoy et al. (2013) re-confirmed the existence of a peculiar south-west clump at approximately $1.4^{\prime \prime}$ to the southwest of the star using LBT/LMIRCam data. Humphreys et al. (2007) studied the total space motions and directions of several features in the envelope of VY CMa. They deduced that the southwest clump is slightly redshifted w.r.t. the systemic velocity and is moving away from us at an angle of $+8^{\circ}$ with a total velocity of only $\sim 18 \mathrm{~km} \mathrm{~s}^{-1}$, of which the transverse component is $\sim 17.7 \mathrm{~km} \mathrm{~s}^{-1}$ and with a negligible radial component of $\sim 2.5 \mathrm{~km} \mathrm{~s}^{-1}$. This derived redshifted behaviour is in accord with the ALMA NaCl flux density shown in Fig. 6 which shows a peak around $25 \mathrm{~km} \mathrm{~s}^{-1}$, or $3 \mathrm{~km} \mathrm{~s}^{-1}$ greater than the stellar velocity of $22 \mathrm{~km} \mathrm{~s}^{-1}$. However, this is not in agreement with the $\mathrm{ALMA} \mathrm{TiO}_{2}$ emission showing up blue shifted (De Beck et al. 2015) (see Sect. 4.2.2).

\section{Discussion}

Metal-bearing molecules are rarely observed in the gas-phase in the ISM due to their large refractory character that makes them 
to easily form solid condensates. Metal halides are among the first metal-containing molecules detected in space. The alkali halide salt molecule, $\mathrm{NaCl}$, was already detected in VY CMa and in the oxygen-rich AGB star IK Tau (Milam et al. 2007; Kamiński et al. 2013b). The high dipole moment of $\mathrm{NaCl}$ is favourable for radiative excitation to play a significant role in populating the (vibra-)rotational levels. For Einstein $A$-values in the range of $0.013-0.019 \mathrm{~s}^{-1}$ (see Table 1) and assuming collision rates within $v=0$ around $10^{-10} \mathrm{~cm}^{3} \mathrm{~s}^{-1}$ (based on the collisional rates of SiS, Agúndez et al. 2012), one gets a critical density $n_{\text {crit }}$ around $2 \times 10^{8} \mathrm{~cm}^{-3}$. Using the output from the thermodynamical model for the wind of VY CMa as presented by Decin et al. (2006), this implies that there is only a small region for $r \lesssim 9 R_{\star}\left(\sim 0.05^{\prime \prime}\right)$ where the gas number density is higher than the critical density and hence where LTE would prevail.

Milam et al. (2007) determined the formation locus of gaseous sodium chloride to be close to the central star, between $\sim 5-40 R_{\star}$, with a fractional abundance relative to $\mathrm{H}_{2}$ of $5 \times 10^{-9}$. This is in accord with thermochemical equilibrium predictions, not taking phase changes into account, predicting that the gaseous $\mathrm{NaCl}$ abundance peaks around 500 to $1200 \mathrm{~K}$ at densities $n \sim 10^{11} \mathrm{~cm}^{-3}$ (see Fig. 3 in Milam et al. 2007), with $\mathrm{NaCl}$ being the main sodium carrier in oxygen-rich environments $^{3}$. For temperatures above $1500 \mathrm{~K}$, the fractional abundance of gaseous $\mathrm{NaCl}$ is below $10^{-12}$. Kamiński et al. (2013b) derived the excitation temperatures and column densities for the molecules detected in their SMA scan of VY CMa from a population diagram analysis, i.e. assuming an isothermal gas in local thermodynamic equilibrium. They determined a column density for $\mathrm{NaCl}$ of $1.3 \pm 0.2 \times 10^{15} \mathrm{~cm}^{-2}$ for a rotational excitation temperature of $210 \pm 25 \mathrm{~K}$; the temperature derived from transitions including vibrationally excited levels is a factor of a few larger and the column density a factor of 2-3. Our source size implies that column densities derived by Kamiński et al. (2013b) should be increased by a factor of $\sim 3$, i.e. $N_{\text {rot }} \approx 5 \times 10^{15} \mathrm{~cm}^{-2}$. A mass loss rate of $10^{-4} M_{\odot} / \mathrm{yr}$ and a velocity of $\sim 15 \mathrm{~km} \mathrm{~s}^{-1}$ (as derived in the $\mathrm{NB}$ ) yields a fractional $\mathrm{NaCl}$ abundance relative to $\mathrm{H}_{2}$ of $\sim 4 \times 10^{-8}$ in the northern blob. We note, however, the very provisional nature of this number. The lack of information on cross sections, physical conditions, radiative pumping efficiency, non-LTE effects, etc. means that extreme caution should be taken when using this value of the column density and $\mathrm{NaCl}$ abundance which is likely uncertain by about a factor of 10 .

The confined distribution of gaseous sodium chloride, the fact that the observed velocities do not reach the terminal velocity, and realising the refractory nature of sodium chloride hint toward the fact that $\mathrm{NaCl}$ condenses easily onto grains. Thermochemical calculations including gas chemistry and the formation of possible liquid and solid condensates predict that sodalite, $\mathrm{Na}_{4}\left[\mathrm{AlSiO}_{4}\right]_{3} \mathrm{Cl}$, is the major silicated chlorine containing condensate in an oxygen-rich environment, while halite, crystalline $\mathrm{NaCl}$ salt, might be formed in a carbon-rich wind (Lodders \& Fegley 1999). However, the fact that the ALMA data prove the existence of gaseous $\mathrm{NaCl}$ at distances far beyond the major dust condensation region, specifically around $1.22^{\prime \prime}$ $\left(\sim 220 R_{\star}\right)$ in the "SW" extension, is an incentive to reconsider the different gas-grain chemical processes at work that might prevent all gaseous $\mathrm{NaCl}$ species to be condensed.

\footnotetext{
3 Based on the output from the thermodynamical model for the wind of VY CMa as presented by Decin et al. (2006), we note that a density of $n \sim 10^{11} \mathrm{~cm}^{-3}$ is reached very close to the stellar atmosphere around $1.02 R_{\star}$, where the gas kinetic temperature is well above $1200 \mathrm{~K}$, i.e. around $2700 \mathrm{~K}$.
}

A simplified $\mathrm{NaCl}$ chemical network is developed in Sect. 4.1 with the aim to constrain the main chemical processes that control the gaseous and solid $\mathrm{NaCl}$ content. Potential geometrical structures as derived from the multi-component $\mathrm{NaCl}$ emission are discussed in Sect. 4.2.

\section{1. $\mathrm{NaCl}$ gas-grain chemistry}

Our aim is to understand the cycling between gaseous and solid $\mathrm{NaCl}$ through the envelope of VY CMa. Since many parameters such as the sticking coefficient, surface binding energy, and the surface density of adsorbing sites are not known, we have constructed a simplified chemical network that lets us control several parameters that directly influence the $\mathrm{NaCl}$ abundance profile. Analogous simplified networks are constructed to study, for instance, the water content in low-mass forming regions (Schmalzl et al. 2014). The main chemical processes to be considered are accretion of gaseous $\mathrm{NaCl}$ onto grains, thermal desorption and photodesorption.

\subsubsection{Simplified $\mathrm{NaCl}$ network}

The rate of accretion of gaseous $\mathrm{NaCl}$ onto existing dust grains is given by

$R_{\mathrm{ACC}}=S \sigma_{\mathrm{d}}\left\langle v_{X}\right\rangle n_{\mathrm{g}}(\mathrm{NaCl})$

in units of $\left[\mathrm{cm}^{-3} \mathrm{~s}^{-1}\right]$, where $S$ is the sticking probability, $\sigma_{\mathrm{d}}$ the total grain surface cross-section per unit volume in units of $\left[\mathrm{cm}^{2} \mathrm{~cm}^{-3}\right],\left\langle v_{X}\right\rangle$ is the average thermal velocity $\sqrt{\left(8 k T_{\mathrm{g}} / m\right)}$ in units of [ $\mathrm{cm} \mathrm{s}^{-1}$ ] with $T_{\mathrm{g}}$ the gas kinetic temperature, $k$ the Boltzmann constant, $m$ the mass of gaseous $\mathrm{NaCl}$ (58.5 atomic mass units), and $n_{\mathrm{g}}(\mathrm{NaCl})$ the number density of gaseous $\mathrm{NaCl}$ in units of $\mathrm{cm}^{-3}$.

We can write the total grain surface cross section per unit volume as

$\sigma_{\mathrm{d}}=\int\left(\pi a^{2}\right) n_{\mathrm{d}}(a, r) \mathrm{d} a$,

with $n_{\mathrm{d}}(a, r)$ being the grain volume density for a grain with radius $a$. We adopt a grain size distribution $n_{\mathrm{d}}(a, r) \mathrm{d} a=$ $K a^{-3.5} n_{\mathrm{H}}(r) \mathrm{d} a$, with a minimum grain size, $a_{\mathrm{min}}$, of $0.005 \mu \mathrm{m}$ and a maximum grain size, $a_{\max }$, of $0.25 \mu \mathrm{m}$. The slope of -3.5 is typical for interstellar grains (Mathis et al. 1977). $n_{\mathrm{H}}$ is the total hydrogen number density in units of $\left[\mathrm{cm}^{-3}\right]$ and $K$ represents an abundance scaling factor giving the number of dust particles in units of particles per $\mathrm{H}$ atom. For the interstellar medium, $K$ is estimated to be around $7.9 \times 10^{-26} \mathrm{~cm}^{2.5} / \mathrm{H}$ for silicate grains (Draine \& Lee 1984). For a dust-to-gas mass ratio of 0.002 (Decin et al. 2006), $K$ is $\sim 2.8 \times 10^{-26} \mathrm{~cm}^{2.5}$ per $\mathrm{H}$ atom (see Appendix B). For the assumed grain size distribution, the total grain surface cross section $\sigma_{\mathrm{d}}$ can hence be written as $\sigma_{\mathrm{d}} \sim 2500 \pi K n_{\mathrm{H}}(r)$ or $\sigma_{\mathrm{d}} \sim 2.2 \times 10^{-22} n_{\mathrm{H}}(r)$.

The number density of gaseous $\mathrm{NaCl}, n_{\mathrm{g}}(\mathrm{NaCl})$, is written as

$n_{\mathrm{g}}(\mathrm{NaCl})(r)=X(\mathrm{NaCl}) n_{\mathrm{H}}(r)$.

Assuming solar elemental abundance of chlorine $\left(\sim 3.2 \times 10^{-7}\right)$ and sodium $\left(\sim 2.1 \times 10^{-6}\right)$ the abundance of $\mathrm{NaCl}, X(\mathrm{NaCl})$, is controlled by the chlorine content. 1982)

The rate of thermal desorption is given by (Tielens \& Hagen

$R_{\mathrm{TD}}=n_{\mathrm{s}}(\mathrm{NaCl}) v_{0} \exp \left(-\mathrm{E}_{\mathrm{b}} / \mathrm{k} \mathrm{T}_{\mathrm{d}}\right)$ 
in units of $\left[\mathrm{cm}^{-3} \mathrm{~s}^{-1}\right]$, where $n_{\mathrm{s}}(\mathrm{NaCl})$ is the number density of $\mathrm{NaCl}$ molecules on the grains per $\mathrm{cm}^{3}, v_{0}$ a characteristic vibration frequency in units of $\left[\mathrm{s}^{-1}\right], E_{\mathrm{b}}$ the surface binding energy, and $T_{\mathrm{d}}$ the dust grain temperature.

To get the characteristic vibration frequency, $v_{0}$, we adopt the harmonic oscillator approach (Hasegawa et al. 1992)

$v_{0}=\sqrt{2 N_{\mathrm{s}} E_{\mathrm{b}} / \pi^{2} m}$,

where $N_{\mathrm{s}}$ is the surface density of adsorbing sites on the grains and $m$ is the mass of the adsorbed $\mathrm{NaCl}$ particle. The number of adsorbing sites per unit area, $N_{\mathrm{s}}$, is assumed to be $\sim 1.5 \times$ $10^{-15} \mathrm{~cm}^{-2}$ (Hasegawa et al. 1992). Hence $v_{0}$ is given by

$v_{0}=2.07 \times 10^{12} \sqrt{\frac{T_{\mathrm{b}}}{10000}}$,

with $T_{\mathrm{b}}$ the surface binding temperature in Kelvin. This is in good accord with the observed frequency of $336 \mathrm{~cm}^{-1}$ (or $1 \times$ $10^{13} \mathrm{~s}^{-1}$ ) listed by Martin (1983) or the value of $380 \mathrm{~cm}^{-1}$ (or $1.13 \times 10^{13} \mathrm{~s}^{-1}$ ) calculated using the Born-Mayer lattice theory by Rittner (1951).

One has to realize that even if all $\mathrm{Cl}$ is in $\mathrm{NaCl}$ and if all $\mathrm{NaCl}$ molecules start off in the gas and accretes onto already formed dust grains, $\mathrm{NaCl}$ will only occupy at most $25 \%$ of the available surface sites, i.e. $\mathrm{NaCl}$ is in the sub-monolayer regime. The reason for that is that the number of sites per unit volume is given by $4 \sigma_{\mathrm{d}} N_{\mathrm{s}}$ or $1.3 \times 10^{-6} n_{\mathrm{H}}(r)$ with the cosmic abundance of $\mathrm{Cl}$ being $3.2 \times 10^{-7} n_{\mathrm{H}}(r)$. As shown by Tielens \& Hagen (1982) quantum mechanical tunneling and thermal hopping of the adsorbed species on the grain surface are not relevant for $\mathrm{NaCl}$.

For sub-monolayer coverage $\mathrm{NaCl}$ can photodesorb from the grain surface through far-ultraviolet (FUV) photons at a rate

$R_{\mathrm{PD}}=\left(F_{\mathrm{FUV}} Y_{\mathrm{PD}} / 4 N_{\mathrm{s}}\right) n_{\mathrm{s}}(\mathrm{NaCl})$,

in units of $\left[\mathrm{cm}^{-3} \mathrm{~s}^{-1}\right] . F_{\mathrm{FUV}}$ is the flux of FUV photons reaching the grain and $Y_{\mathrm{PD}}$ the photodesorption yield.

The photodesorption yield $Y_{\mathrm{PD}}$ is difficult to assess. For grains for which water ice is the dominant mantle component, water molecules are photodesorbed either through the excess energy caused by the recombination of $\mathrm{H}$ and $\mathrm{OH}$ radicals following direct photodissociation or via the "kick-out" mechanism where an excited $\mathrm{H}$ atom ejects an $\mathrm{H}_{2} \mathrm{O}$ molecule upon collision (Arasa et al. 2015). In VY CMa Shenoy et al. (2013) derive a grain temperature of $\sim 130-170 \mathrm{~K}$ at the "SW" clump, making it marginal whether ice mantles can be present - thermal desorption of water occurs at $160 \mathrm{~K}$ (Collings et al. 2004). If no water ice is present, the yield is likely to be much less than $3 \times 10^{-3}$ which is a typical value for molecules in water ice (Öberg et al. 2009a,b; Westley et al. 1995)

Although it is commonly thought that the internal FUV radiation field of RSG stars is negligible, this is not necessarily the case for material sitting close to the stellar photosphere. To calculate the flux at the "SW" clump, we use an effective stellar temperature, $T_{\star}$, of $3480 \mathrm{~K}$, the photodissociation crosssections of $\mathrm{NaCl}$ measured by Silver et al. (1986), and a distance of $250 R_{\star}$ to the "SW" clump. For wavelengths shorter than $2910 \AA$, the threshold wavelength for $\mathrm{NaCl}$ photodissociation, we find that the total FUV flux is roughly $10^{4}$ times larger than the general ISM flux (Draine 1978) and calculate the unshielded photodissociation rate to be $3.2 \times 10^{-5} \mathrm{~s}^{-1}$, which is a lower limit to the rate given that the cross-section has not been measured below $1890 \AA$ and some $10^{4}$ times larger than the unshielded ISM rate (van Dishoeck 1988).
The change of the gaseous and solid $\mathrm{NaCl}$ number densities is then given by

$\frac{\mathrm{d} n_{\mathrm{g}}(\mathrm{NaCl})}{\mathrm{d} t}=-\frac{\mathrm{d} n_{\mathrm{s}}(\mathrm{NaCl})}{\mathrm{d} t}=-R_{\mathrm{ACC}}+R_{\mathrm{TD}}+R_{\mathrm{PD}}$.

and we make the assumption that

$X(\mathrm{Cl}) n_{\mathrm{H}}(r)=n_{\mathrm{g}}(\mathrm{NaCl})+n_{\mathrm{s}}(\mathrm{NaCl})$

at any time $t$ with $X(\mathrm{Cl})$ the cosmic abundance of chlorine relative to hydrogen.

\subsubsection{Chemical network model results}

The value for the surface binding energy, $T_{\mathrm{b}}$, is not known. It depends on the polarizability of $\mathrm{NaCl}$ and its ability to form specific bonds (Tielens \& Hagen 1982) to the surface. In the case of $\mathrm{H}_{2} \mathrm{O}$ with its strong dipole moment and its ability to from hydrogen bonds, the surface binding energy on an ice surface has been calculated to be about $4000 \mathrm{~K}$ (Hale et al. 1981). On the other hand, the surface binding energy of $\mathrm{Na}$ to a $\mathrm{NaCl}$ polycrystalline target is about $1.3 \mathrm{eV}$ (or $\sim 15000 \mathrm{~K}$ ). Considering this, we have decided to study the evolution of the $\mathrm{NaCl}$ abundance for three different values of the surface binding energies: $5000 \mathrm{~K}$, $10000 \mathrm{~K}$, and $15000 \mathrm{~K}$.

It is instructive to follow the abundance of gaseous and solid $\mathrm{NaCl}$ by solving Eq. (7) for $n_{\mathrm{s}}(\mathrm{NaCl})$. Using conservation of $\mathrm{NaCl}$ at any time $t$ (Eq. (8)) and writing $\sigma_{\mathrm{d}}(r)=2.2 \times$ $10^{-22} n_{\mathrm{H}}(r)=\sigma n_{\mathrm{H}}(r)$, the rate equation can be rewritten as

$\frac{\mathrm{d} n_{\mathrm{s}}(\mathrm{NaCl})}{\mathrm{d} t}=a-b n_{\mathrm{s}}(\mathrm{NaCl})$,

where $a=S \sigma n_{\mathrm{H}}^{2} v_{X} X(\mathrm{Cl})$ and $b=S \sigma n_{\mathrm{H}} v_{X}+v_{0} \exp \left(-T_{\mathrm{b}} / T_{\mathrm{d}}\right)+$ $\left(F_{\mathrm{FUV}} Y_{\mathrm{PD}} / 4 N_{\mathrm{s}}\right)$. This has a simple time-dependent solution

$n_{\mathrm{s}}(\mathrm{NaCl}, t)=\frac{a}{b}(1-\exp (-b t))$.

$n_{\mathrm{s}}(\mathrm{NaCl}, t=0)=0$ and the time taken to reach steady state is a few times $1 / b$. The steady-state solution is $n_{\mathrm{s}}(\mathrm{NaCl}, \mathrm{ss})=a / b$ and is relatively insensitive to the value of $S$ for $S \sim 1$.

It is difficult to determine the product $F_{\mathrm{FUV}} Y_{\mathrm{PD}}$ with any degree of certainty. The flux of FUV photons at the "SW" clump is very large if there is no extinction due to dust along the line-ofsight to the star. If this really is the case, then $\mathrm{NaCl}$ is photodesorbed completely on a very fast time-scale and the $\mathrm{NaCl}$ released from the dust will be photodissociated rapidly, on a time-scale of a few days. In this case, $\mathrm{NaCl}$ is very unlikely to reform in the gas phase given that both $\mathrm{Na}$ and $\mathrm{Cl}$ are very minor elements. This scenario is unlikely, however, as the dust condensation radius lies much closer to the star, on the order of 35-55 mas, see Sect. 3.3. Using our derived dust-to-gas mass ratio, we calculate that the photosphere is hidden by some $10^{4}$ mag of visual extinction. We note, however, that even 10 mag of visual extinction is enough to reduce the unshielded FUV flux to interstellar values. Thus stellar FUV photons are unlikely to play a role in $\mathrm{NaCl}$ chemistry.

It is instructive, though, to consider a calculation for which the value of $F_{\mathrm{FUV}} Y_{\mathrm{PD}}=3 \times 10^{5}$, the typical interstellar value. Assuming a sticking coefficient, $S$, of 1 , the steady-state solution for the gaseous and solid $\mathrm{NaCl}$ fractional abundances (with respect to the hydrogen number density) are shown in Fig. 8 for different values of the binding energy. The hydrogen number density in Fig. 8 is taken to be $1 \times 10^{8} \mathrm{~cm}^{-3}$. This value is derived 
T_bind $=5,000 \mathrm{~K}$

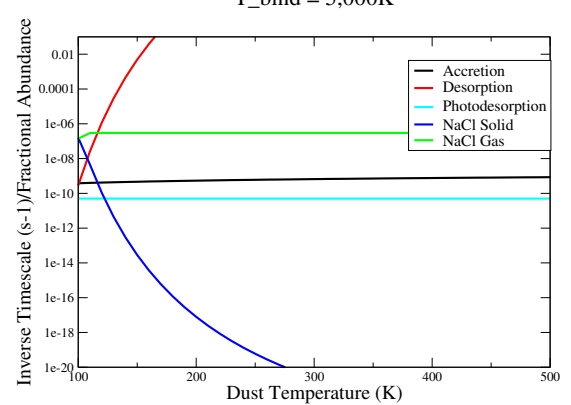

T_bind $=10,000 \mathrm{~K}$

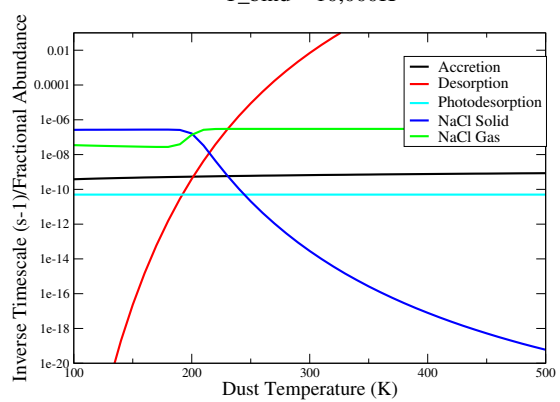

T_bind $=15,000 \mathrm{~K}$

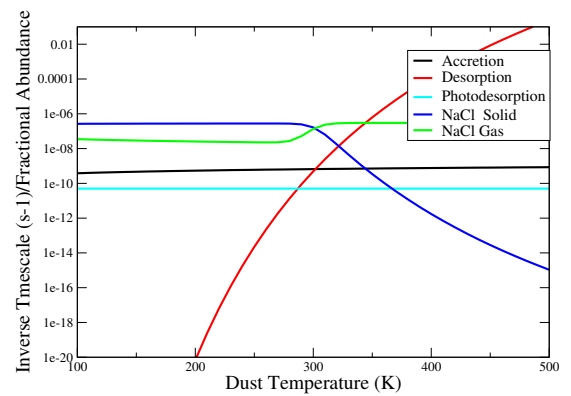

Fig. 8. Steady state solutions for the gaseous and solid $\mathrm{NaCl}$ abundances (relative to the hydrogen number density) as a function of the dust temperature, $T_{\mathrm{d}}$, for the case in which accretion, thermal desorption and photodesorption are included in the chemical network. The different panels are for different values of the binding temperature $(5000,10000,15000 \mathrm{~K})$ at a hydrogen number density of $1 \times 10^{8} \mathrm{~cm}^{-3}$. Each panel also shows the inverse timescale for accretion, thermal desorption and photodesorption in units of $\mathrm{s}^{-1}$, calculated with $F_{\mathrm{FUV}} Y_{\mathrm{PD}}=3 \times 10^{5}$.
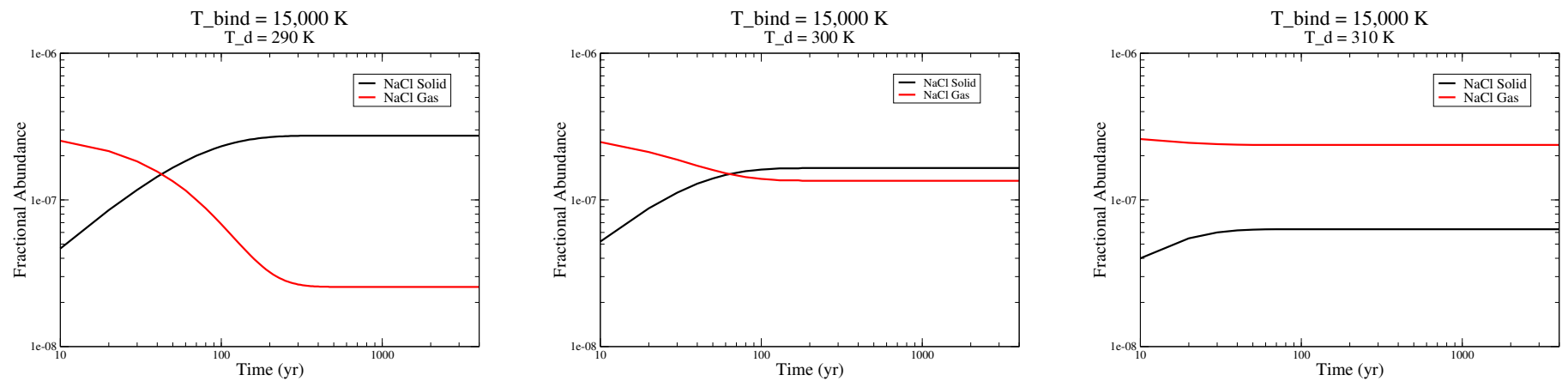

Fig. 9. Fractional abundances of gaseous and solid $\mathrm{NaCl}$ in function of time for a binding temperature of $15000 \mathrm{~K}$ at three different values of the dust temperature $T_{\mathrm{d}}(290,300,310 \mathrm{~K})$.

from the equation of mass conservation $\dot{M}=4 \pi r^{2} \rho_{\mathrm{g}}(r) v_{\mathrm{g}}(r)$, with $\rho_{\mathrm{g}}$ the gas number density and $v_{\mathrm{g}}(r)$ the gas velocity. Using a mass-loss rate $\dot{M}$ of $1 \times 10^{-4} M_{\odot} \mathrm{yr}^{-1}$ and the gas velocity structure as derived from solving the momentum equation (see Decin et al. 2006), a hydrogen number density of $1 \times 10^{8} \mathrm{~cm}^{-3}$ is reached at $\sim 10 R_{\star}$, i.e. the dust condensation locus of interest to this study. Figure 8 also displays the inverse timescale for accretion and thermal desorption. Figure 8 shows that photodesorption occurs at a slower rate than accretion and that only about $10 \%$ of the $\mathrm{NaCl}$ abundance resides in the gas phase at low dust temperatures (relative to the surface binding temperature). For example, for a binding energy of $10000 \mathrm{~K}$, once the grain becomes warm enough, around $200 \mathrm{~K}$, all solid-state $\mathrm{NaCl}$ is thermally desorbed into the gas phase. It is clear from these simulations that the crucial factor determining the amount of solid versus gaseous $\mathrm{NaCl}$ is the ratio of the binding energy to the dust temperature, which appears in Eq. (3) for the thermal desorption. For a binding temperature of $5000 \mathrm{~K}$ all $\mathrm{NaCl}$ will be gaseous unless the dust temperature is below $\sim 120 \mathrm{~K}$. A higher binding energy implies that the dust grains may attain higher temperatures before thermal desorption starts to dominate over the accretion rate. The turn-over point is around $T_{\mathrm{b}} / T_{\mathrm{d}} \sim 50$ (see also Fig. 9). Close to this value, steady state solutions occur on timescales of 50-100 yr as shown in Fig. 9.

At the dust condensation locus of $\sim 10 R_{\star}$, the grain temperatures might be in the range of $500-1000 \mathrm{~K}$, so that all $\mathrm{NaCl}$ will be in gaseous form independent of the assumed binding energy. This is in line with the ALMA observations presented in this paper. A steady state solution of $\sim 100 \mathrm{yr}$ for a velocity of $\sim 15 \mathrm{~km} \mathrm{~s}^{-1}$ (as derived from the ALMA NaCl data) yields a travel distance of $30 R_{\star}$ (or 160 mas). When the grain temperatures becomes lower than $300 \mathrm{~K}$, solid $\mathrm{NaCl}$ starts to form from accretion onto existing grains. Whenever $T_{\mathrm{d}}<100 \mathrm{~K}$, another chemical process than thermal desorption needs to be invoked for $\mathrm{NaCl}$ to remain (partly) in gaseous form. The ALMA data clearly show gaseous $\mathrm{NaCl}$ emission around $1.22^{\prime \prime}$ in the south-western extension, at a place where also scattering by dust grains peaks (see Fig. 5). Assuming only direct stellar radiation, Shenoy et al. (2013) derived a radiative equilibrium temperature for the grains at that distance of $\sim 130-170 \mathrm{~K}$. If the grains indeed have that temperature at $1.22^{\prime \prime}$, this would imply that either the binding temperature is around $5000 \mathrm{~K}$ so that $\mathrm{NaCl}$ stays gaseous that far away from the star or that, in the case of higher values for the binding temperature, another process is important. One possibility is shock-induced sputtering driven by localised mass ejections (see Sect. 4.2.2. Such a process could lead, for example, to the different spatial distributions of refractory molecules, such as observed for $\mathrm{NaCl}$ and $\mathrm{TiO}_{2}$, since their sputtering rates likely depend on their binding energies.

\subsection{Morphology}

The $\mathrm{NaCl}$ emission does not peak at the stellar position "VY", which can be explained by the presence of a density cavity close to the star, where the temperature is too high for gaseous $\mathrm{NaCl}$ to exist in high enough amounts (see previous sections). The morphology in the channel maps is clearly inconsistent with a spherical, uniformly expanding, envelope, which would exhibit a circular or ring morphology with decreasing radius for increasing velocity difference w.r.t. systematic velocity. Previous studies hint toward a preferred axis of symmetry oriented northeastsouthwest at a position angle of $\sim 50^{\circ}$ (see Sect. 1). The central waist in the integrated $\mathrm{NaCl}$ intensity map aligns with the central waist seen in the dust continuum map and, as seen in Figs. 4 
and 5, the ALMA data also roughly support the idea of a preferred axis oriented northeast-southwest.

Deducing the morphology of the circumstellar environment of VY CMa is not a straightforward task. However, the ALMA $\mathrm{NaCl}$ data seem to favour two types of geometries: (1) an axisymmetric geometry, be it a disk, a bipolar outflow, or in general a kind of toroidal overdensity or (2) localized mass ejections. We consider the merits of both geometries regarding the morphology seen in the ALMA data and other optical, infrared and maser data published in the literature.

\subsubsection{Axisymmetric geometry}

Within $1^{\prime \prime}$ of the central star, the ALMA $\mathrm{NaCl}$ data suggest an axial symmetry with a preferred axis oriented NE/SW at a PA of $\sim 50^{\circ}$ (defined North-East), showing both a bright $\mathrm{NaCl}$ and dust continuum peak to either side of the symmetry axis. This kind of spatial-kinematic structure can be interpreted as an equatorially enhanced density structure with the polar axis, hence lower density region, along the NE/SW axis at PA of $\sim 50^{\circ}$.

Different studies (see Sect. 1 and below) support the axisymmetry revealed by the ALMA data. Herbig (1970) invoked a flared disk or ring in latitudes $10^{\circ}$ to $30^{\circ}$ and seen almost edgeon to explain the infrared energy distributions. Using optical and near- and mid-infrared data Smith et al. (2001) gave support to that interpretation and qualitatively interpreted the morphological structure as being caused by an equatorial density enhancement with a bipolar axis projected roughly northeast-southwest, and with the southwest lobe closer to us. The lack of optical and near-infrared emission in the northeast region of their data could be explained by high extinction along the line-of-sight and inefficient backscattering of the light by the dust particles. The SMA data of ${ }^{12} \mathrm{CO}$ and ${ }^{13} \mathrm{CO}$ at a spatial resolution of $\sim 2^{\prime \prime}$ were modelled by Muller et al. (2007) using a dense, compact and dusty central component, embedded in a more diffuse extended envelope and with a high-velocity bipolar outflow oriented in the east-west direction with a wide opening angle $\left(\sim 120^{\circ}\right)$ viewed close to the line-of-sight $\left(i=15^{\circ}\right)$. The inner radius of the bipolar lobes was determined at $\sim 0.6^{\prime \prime}$ and the velocity in the bipolar lobes increased linearly from $15 \mathrm{~km} \mathrm{~s}^{-1}$ to $45 \mathrm{~km} \mathrm{~s}^{-1}$ at the outer radius. An enhanced density shell within the bipolar lobes was needed to fit the observed $\mathrm{CO}$ data.

Two position-velocity (PV) diagrams of the $\mathrm{NaCl} 312 \mathrm{GHz}$ ALMA observations are shown in Fig. 10; the panel to the left (PV1) gives the PV diagram for a slit oriented along the presumed polar/rotation axis at a PA of $50^{\circ}$ to the north-east and the panel to the right shows the PV diagram (PV2) for a slit perpendicular to the polar/rotation axis, i.e. along the presumed equatorial plane.

PV1 diagram is characterized by a S-type signature. The northern clump is the main contributor to the bright peak around $24 \mathrm{~km} \mathrm{~s}^{-1}$ (see middle panel in Fig. 10), while both the northern $\left(\mathrm{NB}_{\text {blue }}\right)$ and southern clump contribute to the blue-shifted peak around $19 \mathrm{~km} \mathrm{~s}^{-1}$. The blue-shifted extension around $14 \mathrm{~km} \mathrm{~s}^{-1}$ arises from $\mathrm{NB}_{\text {blue }}$, while $\mathrm{NB}_{\text {red }}$ explains the extension toward velocities of $\sim 35 \mathrm{~km} \mathrm{~s}^{-1}$. It is tempting to interpret the two horizontal ridges at $\Delta v \sim \pm 2.5 \mathrm{~km} \mathrm{~s}^{-1}$ from the systemic velocity as arising from a disk (or in general a density enhanced equatorial region) inclined to the line-of-sight (hereafter referred to as the low-velocity component; see left panel in Fig. 10). The disk has a projected velocity around $2.5 \mathrm{~km} \mathrm{~s}^{-1}$. The blue-shifted part of the disk is to the south-west, and the red-shifted part to the north-east. There is also a more or less vertical ridge extending to $\pm 15 \mathrm{~km} \mathrm{~s}^{-1}$ w.r.t. the systemic velocity, with 2 other horizontal ridges at $\Delta v \sim \pm 9 \mathrm{~km} \mathrm{~s}^{-1}$ from the systemic velocity (i.e. at 14 and $31 \mathrm{~km} \mathrm{~s}^{-1}$ ). This medium-velocity component ${ }^{4}$ is only seen in the northern clump, with the blue-shifted part slightly more to the north-east than the red-shifted part, i.e. an orientation opposite to the low-velocity disk. This tentatively hints towards an interpretation that the medium-velocity component is a biconical polar outflow, with wide opening angle, perpendicular to the equatorial disk plane. An analogous morphology was also deduced for the carbon star V Hya by (Hirano et al. 2004) and the S-type AGB star $\pi^{1}$ Gru by Chiu et al. (2006) from the analysis of ${ }^{12} \mathrm{CO} J=2-1$ SMA data, showing similar PV diagrams as PV1. With the intensity of "NB" being brighter around $31 \mathrm{~km} \mathrm{~s}^{-1}$ than at $14 \mathrm{~km} \mathrm{~s}^{-1}$, this suggests that the far-side of the bipolar outflow is situated to the north. This orientation supports the analysis by Smith et al. (2001), who suggested that the far side of the optical/near-infrared nebula is situated to the northeast, while the southwest lobe is closer to us. A geometry with an equatorial density enhancement roughly northwest-southeast and with the polar lower density region oriented along a NE/SW axis can also explain the morphology of the dust continuum as seen in the ALMA data and shown in Fig. 2 (O'Gorman et al. 2015).

However, one then might wonder why the medium-velocity wind (aka bipolar outflow) is only seen in the northern clump (see also PV2), if the southwest lobe is closer to us. In the case of optically thin emission, it might be that the accumulation of optical thickness along the line sight is too low when reaching the southern clump and that the sensitivity of the present ALMA data is not high enough to trace these low intensities. Another suggestion is that for one reason or another (see Sect. 4.1) gaseous $\mathrm{NaCl}$ condenses rapidly in the southern lobe and that its abundance is too low to be traced.

However, this axisymmetric geometry can not explain the full morphological picture of the envelope around VY CMa. As clearly shown by the ALMA detection of $\mathrm{NaCl}$ emission in the "SW" clump and as already mentioned in this paper, there are convincing indications of localised density structures that might be caused by randomized mass ejections from the illdefined convective surface of the star. However, even in that case, the proposed lower density polar regions in the northeastsouthwest direction might explain why we see the emission from the $\mathrm{NaCl}$ clump at $1.2^{\prime \prime}$ to the southwest: this clump, that might be caused by a higher density mass-loss event, can receive more illumination from the central star through the low density region which will enhance its excitation. This effect was also invoked by De Beck et al. (2015) to explain the detection of $\mathrm{TiO}_{2}$ in the "SW" clump.

\subsubsection{Localized mass ejections}

The present ALMA $\mathrm{NaCl}$ data can also be geometrically interpreted as showing evidence for localized mass ejections. Episodic mass loss events were already suggested by, e.g. Ziurys et al. (2007) and Smith et al. (2009); see Sect. 1. As described in Sect. 3.2, the flux density of the "NB" shows two local maxima, one around $14 \mathrm{~km} \mathrm{~s}^{-1}$ ("NB blue") and one around

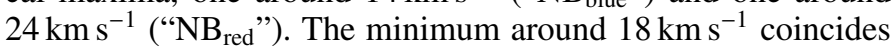
with the maximum in flux density of the southern clump ("SB"). Careful inspection of the channel maps in Fig. 2 also shows that

4 Wind speeds up to $\sim 45 \mathrm{~km} \mathrm{~s}^{-1}$ are present in the nebula surrounding VY CMa (Decin et al. 2006; Smith 2004; Muller et al. 2007). We therefore opt to use "low velocity" for $v<5 \mathrm{~km} \mathrm{~s}^{-1}$ and "medium velocity" for $5<v<15 \mathrm{~km} \mathrm{~s}^{-1}$. 
(Jy/beam)

$\begin{array}{llllll}0 & 0.02 & 0.04 & 0.06 & 0.08 & 0.1\end{array}$

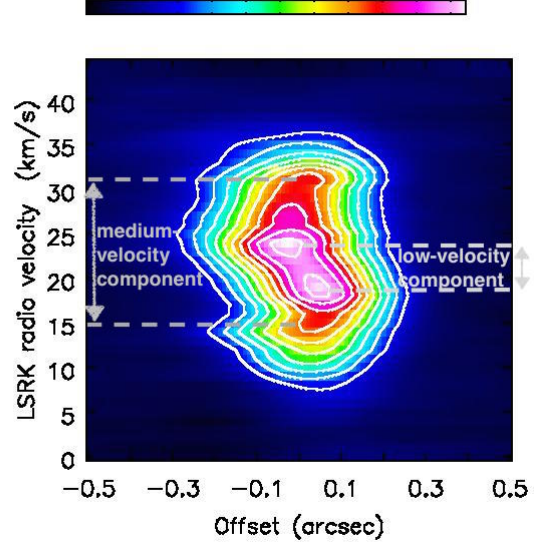

(Jy/beam)

$\begin{array}{llllll}0 & 0.02 & 0.04 & 0.06 & 0.08 & 0.1\end{array}$

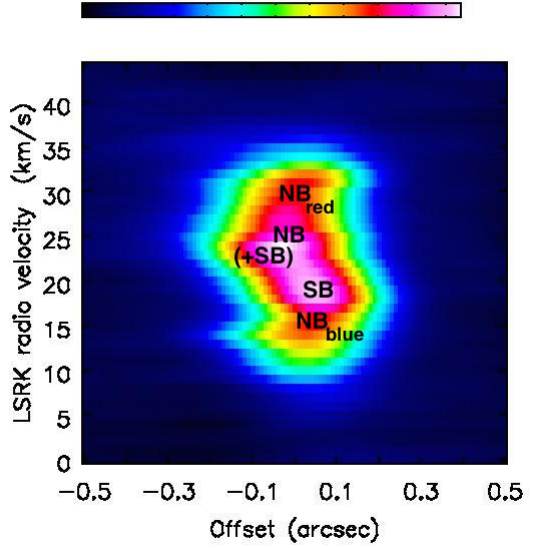

(Jy/beam)

$\begin{array}{llll}a & 0.02 & 0.06 & 0.1\end{array}$

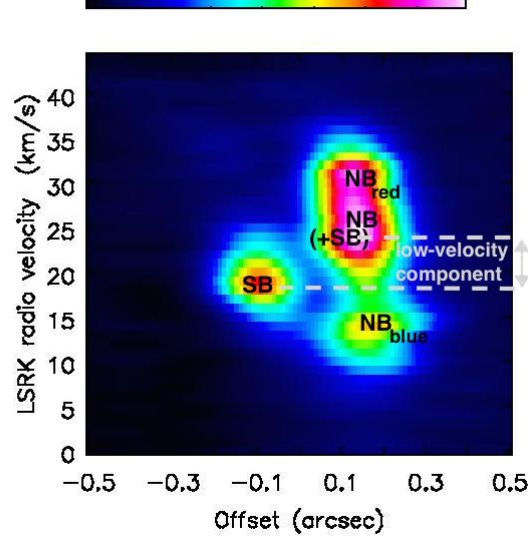

Fig. 10. Position-velocity diagram of the $\mathrm{NaCl} 312 \mathrm{GHz}$ for an axis length of $1^{\prime \prime}$ and an averaging width of $650 \mathrm{mas}$ centred at $\alpha=07 \mathrm{~h} 22 \mathrm{~m} 58.332 \mathrm{~s}$ and $\delta=-25^{\circ} 46^{\prime} 03^{\prime \prime} .053$. In the left and middle panels, the slit has a PA of $50^{\circ}$ (i.e. along the presumed polar/rotation axis; with north-east positions having negative offsets and south-west positions positive offsets), in the right panel the slit PA is $140^{\circ}$ (i.e. along the presumed equatorial plane; with south-east positions having negative offsets and north-west positions positive offsets). In the left and right panels the different velocity components are indicated, while in the middle and right panels the main contributors to the different intensity peaks are indicated. The contours in the left panel are at $[1,2, \ldots 10] \times 0.01 \mathrm{Jy} /$ beam.

(albeit barely spatially resolved) the morphology of "NB blue" has a more pronounced north-south extension than " $\mathrm{NB}_{\text {red }}$ ". While the $\mathrm{TiO}_{2}$ emission coinciding with the "SW" clump is blueshifted (De Beck et al. 2015), the $\mathrm{NaCl}$ channel maps clearly show that the main emission component in this case is slightly red-shifted. The spatially distinct clumps at different projected velocities might hence be interpreted as tracers of different localized mass ejection events, and it is even plausible that " $\mathrm{NB}_{\text {blue" }}$ marks an event different from "NB red". Some of these events are heading away from the observer, other ones are approaching us. This type of 3-dimensional geometry has already been suggested in the literature (see Sect. 1). Combining visual and near-IR images and $4.6 \mu \mathrm{m}$ CO spectra Smith et al. (2009) proposed a geometry where a halo is responsible for an ubiquitous $25 \mathrm{~km} \mathrm{~s}^{-1}$ outflow with potentially mild axisymmetry. Faster $\left(\sim 40 \mathrm{~km} \mathrm{~s}^{-1}\right)$ dense $\mathrm{CO}$ cloudlets move through this halo and create a very asymmetric envelope. The $\mathrm{NaCl}$ clumps clearly trace much lower velocities, albeit their emission is detected far beyond the dust formation radius estimated to be around $10 R_{\star}$ (or 55 mas Monnier et al. 1999). We can not exclude the coincidental fact that the different $\mathrm{NaCl}$ clumps have space vectors quite close to the plane of the sky. However, it might be that these clumps indeed trace lower velocity structures. We note that Kamiński et al. (2013a) also deduced a velocity around $15 \mathrm{~km} \mathrm{~s}^{-1}$ for the "SW" clump, but other molecules in the SMA survey which emit closer to the central target trace velocities larger than the ALMA $\mathrm{NaCl}$ "NB" and "SB" clumps. In the case that the $\mathrm{NaCl}$ clumps and $\mathrm{CO}$ cloudlets are part of the same physical structure, the interaction interface between the cloudlet and the surrounding material could enhance the gas temperature thus facilitating the excitation of both $\mathrm{CO}$ and $\mathrm{NaCl}$.

In the case of localized mass ejections, each event will have more or less the same chemical content at the moment of its ejection and the same laws of thermodynamics and chemistry will apply to each of them. One then might wonder why the morphology as seen in $\mathrm{NaCl}$ is so different compared to what is traced by the ALMA $\mathrm{TiO}_{2}$ channel maps presented by De Beck et al. (2015), a molecule that also has a high dipole moment (6.33 Debye) and with an expected abundance slightly higher than $\mathrm{NaCl}$ in the inner wind region (Gobrecht et al. 2016).
We simply note here that the Grotrian diagram of $\mathrm{NaCl}$ is less complex than for $\mathrm{TiO}_{2}$ and that even different $\mathrm{TiO}_{2}$ lines with similar excitation energies show quite different channel maps. This leads us to propose that the different mass ejections have very short dynamical times and different molecules/excitations might (partly) trace different events where the local thermodynamical, radiative and chemical properties are different. The nature of the star led Smith et al. (2001) to suggest that these ejection events could be of convective or magnetic origin, alike the Sun with its solar flares and eruptions.

There is, however, some similarity between the ALMA NaCl and $\mathrm{TiO}_{2}$ emission, i.e. both molecules trace the "SW" clump out to $\sim 1.2^{\prime \prime}$, a clump also detected in SiS, CS, NS, and $\mathrm{H}_{2} \mathrm{~S}$ in the SMA data of Kamiński et al. (2013a) and in dust scattered light out to $1.4^{\prime \prime}$ (Smith et al. 2001; Shenoy et al. 2013). Some of these molecules are expected to be quite abundant (as SiS) and others not, and they have very different dipole moments (from 1 to 9 Debye). This might indicate that they trace the same physical event along a northeast-southwest axis. The "SW" clump is potentially embedded within the lower density southwest bipolar lobe (see Sect. 4.2.1) or it might be that the dust clump is a high mass-loss event leaving a lower density wake behind it. In both cases, the lower density facilitates the radiative excitation of the molecules by direct stellar light. And while the nature of the bright "C" component in the ALMA dust continuum map is not yet clear (O' Gorman et al. 2015), in retrospective it might be that this dust clump also swept up material while moving away from the star leaving a lower density region behind it that facilitates the excitation of $\mathrm{NaCl}$ seen in the "SB", this under the assumption that both are in the same direction w.r.t. the central star.

\section{Conclusions}

In this paper we have analysed gaseous $\mathrm{NaCl}$ in the inner wind of the luminous red supergiant VY CMa. The high sensitivity and high spatial resolution capabilities of ALMA allow a detection of this molecule within $250 R_{\star}$ from the central star. Four $\mathrm{NaCl}$ lines are detected, being the $v=0,1 J=24-23$ and $J=25-24$ rotational lines in ALMA band 7 at a spatial 
resolution of $\sim 0^{\prime} ! 24 \times 00^{\prime \prime} 13$. The ALMA data permit the first spatial decomposition of the $\mathrm{NaCl}$ emission in up to four different spatial regions. We show that all $\mathrm{NaCl}$ lines show a bimodal spatial distribution, with a bright emission component to the north of the central star and a slightly weaker emission component to the south at an average separation of 100 mas (or $\sim 18 R_{\star}$ ) and 190 mas (or $\sim 34 R_{\star}$ ), respectively. The rotational $\mathrm{NaCl}$ lines in the ground vibrational state show in addition an extra emission component at $\sim 1.22^{\prime \prime}$ to the southwest of the star. This southwest clump is already detected in other molecules (CS, SiS, NS and $\mathrm{H}_{2} \mathrm{~S}$ ) with the SMA (Kamiński et al. 2013a) and in $\mathrm{TiO}_{2}$ with ALMA (De Beck et al. 2015) and is aligned with the southwest extension seen in dust scattered light (Smith et al. 2001) and other near-infrared and polarimetric data (e.g. Monnier et al. 1999; Jones et al. 2007).

The $\mathrm{NaCl}$ lines are detected up to a velocity of $17 \mathrm{~km} \mathrm{~s}^{-1}$ w.r.t. the local standard of rest velocity, $v_{\mathrm{LSR}}$, of $22 \mathrm{~km} \mathrm{~s}^{-1}$. This is significantly lower than the terminal velocity, which is around $35 \mathrm{~km} \mathrm{~s}^{-1}$ as estimated from low- $J$ rotational CO lines. This low velocity was already noticed by Milam et al. (2007), who explained this as being due to the very efficient condensation of $\mathrm{NaCl}$, being a highly refractory species. However, the fact that we detect gaseous $\mathrm{NaCl}$ at distance of $1.2^{\prime \prime}$, far beyond the main dust formation region estimated around 35-55 mas $\left(\sim 10 R_{\star}\right)$, points toward a chemical process preventing all gaseous $\mathrm{NaCl}$ from nucleating. We construct a simplified chemical network aiming at understanding the cycling between gaseous and solid $\mathrm{NaCl}$ through the envelope of $\mathrm{VY} \mathrm{CMa}$. We show that at the dust condensation locus where the grain temperatures can be above $300 \mathrm{~K}$, thermal desorption dominates the rate of accretion of gaseous $\mathrm{NaCl}$ onto existing dust grains. For grain temperatures lower than $100 \mathrm{~K}$, photodesorption is unlikely to be important and removal of $\mathrm{NaCl}$ from the dust is likely due to shock sputtering driven by localized mass ejection events.

It is not straightforward to interpret the geometrical signatures in the inner wind. Based on the ALMA data, and previous optical, near-IR, and mid-IR data two scenarios are favoured. In the first scenario, the overall density structure in the inner wind is described by an equatorial density-enhanced region with the lower density bipolar regions oriented northeast-southwest at a position angle of $\sim 50^{\circ}$. The density-enhanced region, be it a disk, has a projected velocity of $2.5 \mathrm{~km} \mathrm{~s}^{-1}$. The far side of the bipolar lobes is situated to the north, in agreement with the non-detection of the optical reflection nebula to the northeast of the star by Smith et al. (2001). However, this picture alone can not explain all morphological signatures. The south-west clump at $1.2^{\prime \prime}$ and the presence of arcs and knots in optical images point toward the action of localised mass ejections creating higher density structures within the diffuse halo. The present data, obtained with only 16-20 ALMA antennas, can not prove the validity of both scenarios, albeit it seems likely that both play a role in shaping the wind of VY CMa.

Acknowledgements. This paper makes use of the following ALMA data ADS/JAO.ALMA2011.0.00011.SV. ALMA is a partnership of ESO (representing its member states), NSF (USA) and NINS (Japan), together with NRC (Canada) and NSC and ASIAA (Taiwan), in cooperation with the Republic of Chile. The Joint ALMA Observatory is operated by ESO, AUI/NRAO and NAOJ.

\section{References}

Adande, G. R., Edwards, J. L., \& Ziurys, L. M. 2013, ApJ, 778, 22 Agúndez, M., Fonfría, J. P., Cernicharo, J., et al. 2012, A\&A, 543, A48
Arasa, C., Koning, J., Kroes, G.-J., Walsh, C., \& van Dishoeck, E. F. 2015, A\&A, 575, A121

Bowers, P. F., Johnston, K. J., \& Spencer, J. H. 1983, ApJ, 274, 733

Chiu, P.-J., Hoang, C.-T., Dinh-V-Trung, et al. 2006, ApJ, 645, 605

Choi, Y. K., Hirota, T., Honma, M., et al. 2008, PASJ, 60, 1007

Collings, M. P., Anderson, M. A., Chen, R., et al. 2004, MNRAS, 354, 1133

Danchi, W. C., Bester, M., Degiacomi, C. G., Greenhill, L. J., \& Townes, C. H. 1994, AJ, 107, 1469

De Beck, E., Vlemmings, W., Muller, S., et al. 2015, A\&A, 580, A36

de Jager, C., Nieuwenhuijzen, H., \& van der Hucht, K. A. 1988, A\&AS, 72, 259

Decin, L., Hony, S., de Koter, A., et al. 2006, A\&A, 456, 549

Draine, B. T. 1978, ApJS, 36, 595

Draine, B. T., \& Lee, H. M. 1984, ApJ, 285, 89

Efstathiou, A., \& Rowan-Robinson, M. 1990, MNRAS, 245, 275

Gobrecht, D., Cherchneff, I., Sarangi, A., Plane, J. M. C., \& Bromley, S. T. 2016, A\&A, 585, A6

Hale, B. N., Kiefer, J., \& Ward, C. A. 1981, J. Chem. Phys., 75, 1991

Hasegawa, T. I., Herbst, E., \& Leung, C. M. 1992, ApJS, 82, 167

Herbig, G. H. 1970, ApJ, 162, 557

Herbig, G. H. 1972, ApJ, 172, 375

Hirano, N., Shinnaga, H., Dinh-V-Trung, et al. 2004, ApJ, 616, L43

Humphreys, R. M., Helton, L. A., \& Jones, T. J. 2007, AJ, 133, 2716

Jones, T. J., Humphreys, R. M., Helton, L. A., Gui, C., \& Huang, X. 2007, AJ, 133, 2730

Josselin, E., \& Plez, B. 2007, A\&A, 469, 671

Kamiński, T., Gottlieb, C. A., Menten, K. M., et al. 2013a, A\&A, 551, A113

Kamiński, T., Gottlieb, C. A., Young, K. H., Menten, K. M., \& Patel, N. A. 2013b, ApJS, 209, 38

Kastner, J. H., \& Weintraub, D. A. 1998, AJ, 115, 1592

Lodders, K., \& Fegley, Jr., B. 1999, in Asymptotic Giant Branch Stars, eds. T. Le Bertre, A. Lebre, \& C. Waelkens, IAU Symp., 191, 279

Martin, T. P. 1983, Phys. Rep., 95, 167

Mathis, J. S., Rumpl, W., \& Nordsieck, K. H. 1977, ApJ, 217, 425

McCarthy, Jr., D. W. 1979, in High Angular Resolution Stellar Interferometry, eds. J. Davis, \& W. J. Tango, IAU Colloq, 50, 18

Milam, S. N., Apponi, A. J., Woolf, N. J., \& Ziurys, L. M. 2007, ApJ, 668, L131

Monnier, J. D., Tuthill, P. G., Lopez, B., et al. 1999, ApJ, 512, 351

Monnier, J. D., Millan-Gabet, R., Tuthill, P. G., et al. 2004, ApJ, 605, 436

Morris, M., \& Bowers, P. F. 1980, AJ, 85, 724

Müller, H. S. P., Thorwirth, S., Roth, D. A., \& Winnewisser, G. 2001, A\&A, 370, L49

Müller, H. S. P., Schlöder, F., Stutzki, J., \& Winnewisser, G. 2005, J. Mol. Struct., 742,215

Muller, S., Dinh-V-Trung, Lim, J., et al. 2007, ApJ, 656, 1109

Nieuwenhuijzen, H., \& de Jager, C. 1990, A\&A, 231, 134

Öberg, K. I., Garrod, R. T., van Dishoeck, E. F., \& Linnartz, H. 2009a, A\&A, 504,891

Öberg, K. I., Linnartz, H., Visser, R., \& van Dishoeck, E. F. 2009b, ApJ, 693, 1209

O'Gorman, E., Vlemmings, W., Richards, A. M. S., et al. 2015, A\&A, 573, L1

Pun, C. S. J., Michael, E., Zhekov, S. A., et al. 2002, ApJ, 572, 906

Reimers, D. 1975, Memoires of the Societe Royale des Sciences de Liege, 8, 369

Richards, A. M. S. 1997, Ph.D Thesis, University of Manchester, UK

Richards, A. M. S., Yates, J. A., \& Cohen, R. J. 1998, MNRAS, 299, 319

Richards, A. M. S., Impellizzeri, C. M. V., Humphreys, E. M., et al. 2014, A\&A, 572, L9

Rittner, E. S. 1951, J. Chem. Phys., 19, 1030

Schmalzl, M., Visser, R., Walsh, C., et al. 2014, A\&A, 572, A8

Shenoy, D. P., Jones, T. J., Humphreys, R. M., et al. 2013, AJ, 146, 90

Silver, J. A., Worsnop, D. R., Freedman, A., \& Kolb, C. E. 1986, J. Chem. Phys., 84,4378

Smith, N. 2004, MNRAS, 349, L31

Smith, N., Hinkle, K. H., \& Ryde, N. 2009, AJ, 137, 3558

Smith, N., Humphreys, R. M., Davidson, K., et al. 2001, AJ, 121, 1111

Tenenbaum, E. D., Dodd, J. L., Milam, S. N., Woolf, N. J., \& Ziurys, L. M. 2010 ApJS, 190, 348

Thirumalai, A., \& Heyl, J. S. 2012, MNRAS, 422, 1272

Tielens, A. G. G. M., \& Hagen, W. 1982, A\&A, 114, 245

van Dishoeck, E. F. 1988, in Rate Coefficients in Astrochemistry, eds. T. J. Millar, \& D. A. Williams, Astrophys. Space Sci. Libr., 146, 49

Westley, M. S., Baragiola, R. A., Johnson, R. E., \& Baratta, G. A. 1995, Planet. Space Sci., 43, 1311

Wittkowski, M., Hauschildt, P. H., Arroyo-Torres, B., \& Marcaide, J. M. 2012, A\&A, 540, L12

Zhang, B., Reid, M. J., Menten, K. M., \& Zheng, X. W. 2012, ApJ, 744, 23

Ziurys, L. M., Milam, S. N., Apponi, A. J., \& Woolf, N. J. 2007, Nature, 447, 1094 
Appendix A: Channel maps of the $\mathrm{NaCl} v=0,1$ $J=25-24$ lines

Figures A.1 and A.2 show the channel maps for the $\mathrm{NaCl} v=0,1$ $J=25-24$ emission lines.

\section{Appendix B: Additional equations}

The dust-to-gas mass ratio, $\psi$, is defined as

$\psi=\frac{n_{\mathrm{d}} m_{\mathrm{d}}}{\rho_{\mathrm{g}}}$

with $n_{\mathrm{d}}(a, r)$ being the grain volume density (see Sect. 4.1), $m_{\mathrm{d}}$ the mass of the grain being $4 / 3 \pi a^{3} \rho_{\mathrm{s}}$, with $\rho_{\mathrm{s}}$ the specific density of the dust grain taken to $3.3 \mathrm{~g} \mathrm{~cm}^{-3}$ for silicate grains (Draine $\&$ Lee 1984), and $\rho_{\mathrm{g}}$ the gas mass density being $n_{\mathrm{H}}(r) m_{\mathrm{H}}$, with $m_{\mathrm{H}}$ the mass of a hydrogen atom. Applying the same grain size distribution as described in Sect. 4.1, it is easily shown that

$\psi=7 \times 10^{22} K$.

For a dust-to-gas mass ratio of 0.002 , this implies that $K$ is equal to $2.8 \times 10^{-26} \mathrm{~cm}^{2.5}$ per $\mathrm{H}$ atom.

The grain density $n_{\mathrm{d}}(r)=\int n(a, r) \mathrm{d} a$ is then given by $2.2 \times$ $10^{15} K n_{\mathrm{H}}(r)$, which for the given value of $K$ results in $n_{\mathrm{d}}(r)=$ $6 \times 10^{-11} n_{\mathrm{H}}(r)$.

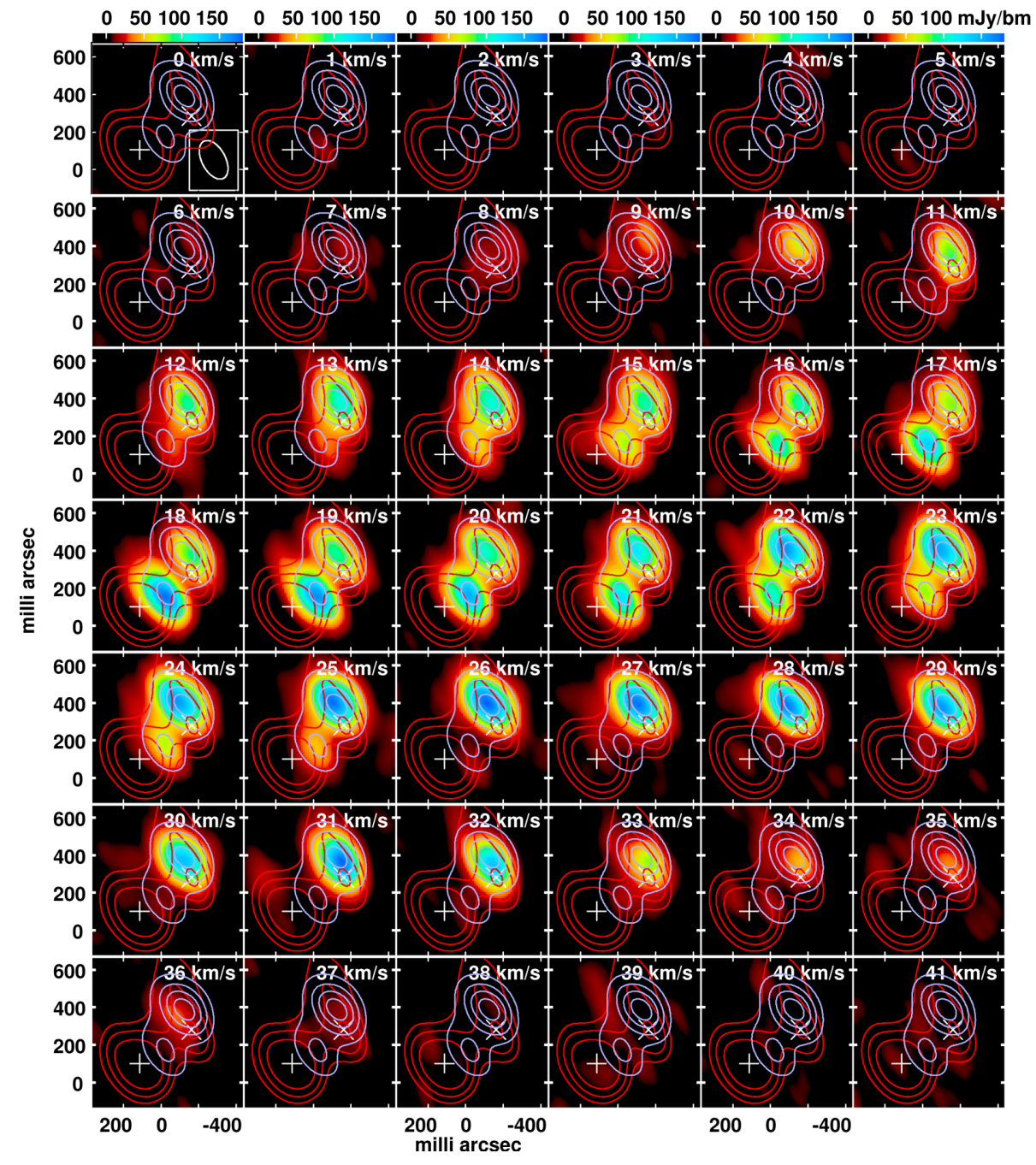

Fig. A.1. Same as Fig. 2 but for the $\mathrm{NaCl} v=1$ $J=25-24$ line at $322 \mathrm{GHz}$. 
$0200400600020040060002004006000200400600 \quad 0200400600 \quad 0200400 \mathrm{mJy} / \mathrm{bm}$

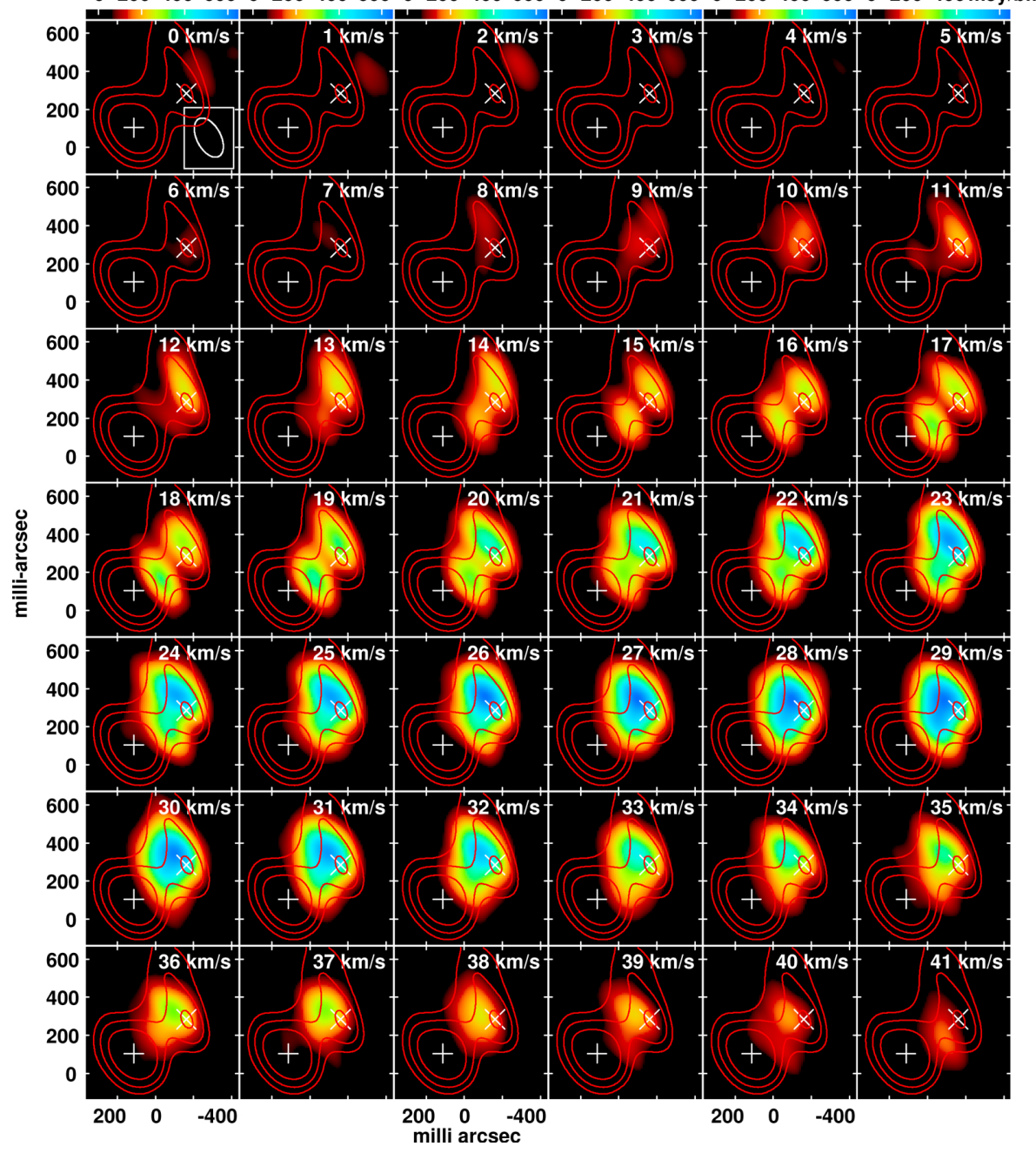

Fig. A.2. Same as Fig. 2 but for the $\mathrm{NaCl} v=0$ $J=25-24$ line at $325 \mathrm{GHz}$. Since this line is

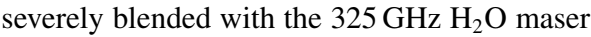
line and the $\operatorname{SiS} v=1 J=18-17$ line the integrated line strength is not shown. 